\title{
GENERAL PROJECTIVE THEORY OF SPACE CURVES*
}

\author{
BY
}

\section{E. J. WILCZYNSKI †}

The theory of the invariants of a linear differential equation is not new. Laguerre, Brioschi, Halphen, Forsyth, Bouton, Fano and others have written important papers on the subject. But in alrnost all of these papers the point of view is essentially analytical. It is only in the brilliant contributions. of HALPHEN that one finds the idea of founding a geometry of curves upon this theory. But even Halphes's papers do not give the ground work for a thorough comprehension of the subject. This is to be found in the geometric interpretation of the semi-covariants which we shall discuss in this paper. With this as a basis, the whole theory becomes clear and transparent, and HaLPHeN's results can easily be connected with it.

I have myself shown in recent years, how the general projective theory of ruled surfaces depends upon the theory of the invariants and covariants of a system of two linear differential equations of the second order. The present paper is governed by similar ideas, and to some extent depends upon this other theory. It is the main purpose of this paper to bring up the general projective theory of curves, based upon the theory of invariants, to the same level of perfection as the corresponding theory of ruled surfaces. Some of the theorems which we shall find, have of course been known for a long time. But even for most of these our proofs will be new.

\section{\$1. The invariants and covoriants.}

We shall confine our attention to the linear differential equation of the fourth order

$$
y^{(4)}+4 p_{1} y^{(3)}+6 p_{2} y^{\prime \prime}+4 p_{3} y^{\prime}+p_{4} y=0
$$

where

$$
y^{\prime}=\frac{d y}{d x}, \quad y^{\prime \prime}=\frac{d^{2} y}{d x^{2}}, \quad \text { etc. }
$$

If we make the transformations

$$
y=\lambda(x) \bar{y}, \quad \xi=\xi(x),
$$

* Presented to the Society (San Francisco) October 1, 1904. Received for publication July 16, 1904.

tOf the Carnegie Institution of Washington.

Trans. Am. Math. Sor. 8 
where $\lambda$ and $\mu$ are arbitrary functions, we obtain another equation of the same form as (1). The functions of the coefficients of (1), which remain invariant under these transformations, are the invariants. If they involve also the functions $y, y^{\prime}, y^{\prime \prime}, y^{(3)}$, they are called covariants.

To determine these invariant functions, it is best according to LIE's theory to make use of infinitesimal transformations. But this has been done in a num. ber of papers by other authors and need not therefore be repeated. We shall, however, need the finite transformations for the later parts of this paper, and shall, therefore, write them down.

Consider first the simpler transformation

$$
y=\lambda(x) \bar{y},
$$

of the dependent variable alone. We shall speak of the corresponding invariant functions as seminvariants and semi-covariants. Let

$$
\bar{y}^{(4)}+4 \pi_{1} \bar{y}^{(3)}+6 \pi_{2} \bar{y}^{\prime \prime}+4 \pi_{3} \bar{y}^{\prime}+\pi_{4} \bar{y}=0
$$

be the transformed equation. Then

$$
\begin{aligned}
& \pi_{1}=\frac{\lambda^{\prime}+p_{1} \lambda}{\lambda}, \\
& \pi_{2}=\frac{\lambda^{\prime \prime}+2 p_{1} \lambda^{\prime}+p_{2} \lambda}{\lambda}, \\
& \pi_{3}=\frac{\lambda^{(3)}+3 p_{1} \lambda^{\prime \prime}+3 p_{2} \lambda^{\prime}+p_{3} \lambda}{\lambda}, \\
& \pi_{4}=\frac{\lambda^{(4)}+4 p_{1} \lambda^{(3)}+6 p_{2} \lambda^{\prime \prime}+4 p_{3} \lambda^{\prime}+p_{4} \lambda}{\lambda},
\end{aligned}
$$

whence one may deduce the absolute seminvariants

$$
\begin{aligned}
& P_{2}=p_{2}-p_{1}^{\prime}-p_{1}^{2}, \\
& P_{3}=p_{3}-p_{1}^{\prime \prime}-3 p_{1} p_{2}+2 p_{1}^{3}, \\
& P_{4}=p_{4}-4 p_{1} p_{3}-3 p_{2}^{2}+12 p_{1}^{2} p_{2}-6 p_{1}^{4}-p_{1}^{(3)},
\end{aligned}
$$

and the relative semi-covariants, besides $y$ which is obviously itself a semicovariant,

$$
\begin{aligned}
& z=y^{\prime}+p_{1} y \\
& \rho=y^{\prime \prime}+2 p_{1} y^{\prime}+p_{2} y, \\
& \sigma=y^{(3)}+3 p_{1} y^{\prime \prime}+3 p_{2} y^{\prime}+p_{3} y .
\end{aligned}
$$

The absolute semi-covariants are $z / y, \rho / y, \sigma / y$. All other semi-covariants and seminvariants are functions of these and of the derivatives of $P_{2}, P_{3}, P_{4}$. 
From (4) we deduce the following equations, which we shall use later:

(5)

$$
\begin{aligned}
& y^{\prime}=-p_{1} y+z, \\
& z^{\prime}=-P_{2} y-p_{1} z+\rho, \\
& \rho^{\prime}=-\left(P_{3}-P_{2}^{\prime}\right) y-2 P_{2} z-p_{1} \rho+\sigma, \\
& \dot{\sigma}^{\prime}=-\left(P_{4}-P_{3}^{\prime}\right) y-3\left(P_{3}-P_{2}^{\prime}\right) z-3 P_{2} \rho-p_{1} \sigma,
\end{aligned}
$$

and also$$
y^{\prime \prime}=\left(2 p_{1}^{2}-p_{2}\right) y-2 p_{1} z+\rho,
$$

$$
y^{(3)}=\left(-p_{3}+6 p_{1} p_{2}-6 p_{1}^{3}\right) y+\left(-3 p_{2}+6 p_{1}^{2}\right) z-3 p_{1} \rho+\sigma,
$$$$
y^{(4)}=\left(-p_{4}+8 p_{1} p_{3}-36 p_{1}^{2} p_{2}+6 p_{2}^{2}+24 p_{1}^{4}\right) y
$$

$$
+\left(-4 p_{3}+24 p_{1} p_{2}-24 p_{1}^{3}\right) z+\left(-6 p_{2}+12 p_{1}^{2}\right) \rho-4 p_{1} \sigma .
$$

We now proceed to make a transformation of the independent variable $\xi=\xi(x)$. We find, denoting the coefficients of the transformed equation by $\bar{p}_{k}$,

$$
\begin{aligned}
& \bar{p}_{1}=\frac{1}{\xi^{\prime}}\left(p_{1}+\frac{8}{2} \eta\right), \\
& \bar{p}_{2}=\frac{1}{\left(\xi^{\prime}\right)^{2}}\left[p_{2}+2 \eta p_{1}+\frac{1}{6}\left(4 \mu+9 \eta^{2}\right)\right], \\
& \bar{p}_{3}=\frac{1}{\left(\xi^{\prime}\right)^{3}}\left[p_{3}+\frac{8}{2} \eta p_{2}+\left(\mu+\frac{3}{2} \eta^{2}\right) p_{1}+\frac{1}{4}\left(\mu^{\prime}+4 \eta \mu+3 \eta^{3}\right)\right], \\
& \bar{p}_{4}=\frac{1}{\left(\xi^{\prime}\right)^{4}} p_{4},
\end{aligned}
$$

where we have put

We find further

$$
\eta=\frac{\xi^{\prime \prime}}{\xi^{\prime}}, \quad \mu=\eta^{\prime}-\frac{1}{2} \eta^{2}
$$

$$
\begin{aligned}
& \bar{z}=\frac{1}{\xi^{\prime}}\left(z+\frac{3}{2} \eta y\right), \\
& \bar{\rho}=\frac{1}{\left(\xi^{\prime}\right)^{2}}\left[\rho+2 \eta z+\frac{1}{6}\left(4 \mu+9 \eta^{2}\right) y\right], \\
& \bar{\sigma}=\frac{1}{\left(\xi^{\prime}\right)^{3}}\left[\sigma+\frac{3}{2} \eta \rho+\left(\mu+\frac{3}{2} \eta^{2}\right) z+\frac{1}{4}\left(\mu^{\prime}+4 \eta \mu+3 \eta^{3}\right) y\right],
\end{aligned}
$$

cogredient with (7). 
Making use of these equations, we find

$$
\begin{aligned}
& \bar{P}_{2}=\frac{1}{\left(\xi^{\prime}\right)^{2}}\left[P_{2}-\frac{5}{6} \mu\right], \\
& \bar{P}_{3}=\frac{1}{\left(\xi^{\prime}\right)^{3}}\left[P_{3}-3 \eta P_{2}-\frac{5}{4} \mu^{\prime}+{ }_{-4}^{10} \eta \mu\right], \\
& \bar{P}_{4}=\frac{1}{\left(\xi^{\prime}\right)^{4}}\left[P_{4}-6 \eta P_{3}-4 \eta^{\prime} P_{2}+11 \eta^{2} P_{2}+\frac{15}{2} \eta \mu^{\prime}-\frac{15}{2} \eta^{2} \mu\right. \\
& \left.-\frac{3}{2} \mu^{\prime \prime}+\frac{19}{6} \mu^{2}\right]
\end{aligned}
$$

whence

$$
\begin{aligned}
& \overline{P_{2}^{\prime}}=\frac{1}{\left(\xi^{\prime}\right)^{3}}\left[P_{2}^{\prime}-2 \eta P_{2}-{ }_{6}^{5} \mu^{\prime}+\frac{5}{3} \eta \mu\right], \\
& \overline{P_{2}^{\prime \prime}}=\frac{1}{\left(\xi^{\prime}\right)^{4}}\left[P_{2}^{\prime \prime}-5 \eta P_{2}^{\prime}-2 \mu P_{2}+5 \eta^{2} P_{2}-\frac{5}{6} \mu^{\prime \prime}+\frac{5}{3} \mu^{2}-\frac{25}{6} \mu \eta^{2}+\frac{25}{6} \mu^{\prime} \eta\right] \\
& \overline{P_{3}^{\prime}}=\frac{1}{\left(\xi^{\prime}\right)^{4}}\left[P_{3}^{\prime}-3 \eta\left(P_{2}^{\prime}+P_{3}\right)-3 \mu P_{2}+\frac{1}{2}-\eta^{2} P_{2}-\frac{5}{4} \mu^{\prime \prime}\right. \\
& \left.+\frac{25}{4}-\eta \mu^{\prime}-\frac{25}{4} \mu \eta^{2}+\frac{10}{4} \mu^{2}\right] \text {. }
\end{aligned}
$$

We find therefore the following invariants and covariants :

$$
\begin{aligned}
\theta_{3} & =P_{3}-\frac{3}{2} P_{2}^{\prime}, \quad \theta_{4}=P_{4}-2 P_{3}^{\prime}+{ }_{5}^{6} P_{2}^{\prime \prime}-\frac{6}{25} P_{2}^{2}, \\
\theta_{3 \cdot 1} & =6 \theta_{3} \theta_{3}^{\prime \prime}-7 \theta_{3}^{\prime 2}-\frac{108}{5} P_{2} \theta_{3}^{2}, \\
C_{2} & =10 z^{2}-15 y \rho-12 P_{2} y^{2}, \\
C_{3} & =10 z^{3}-3 C_{2} z-9\left(5 \sigma+6 P_{2} z+P_{3} y\right) y^{2}, \\
C_{4} & =2 \theta_{3} z+\theta_{3}^{\prime} y,
\end{aligned}
$$

where the index indicates the weight. In denoting one invariant of weight 8 by $\theta_{3.1}$ we follow a notation due to Forsyth. An invariant may be regarded as a covariant of degree zero. With this understanding, it suffices to say that the effect of the complete transformation

$$
\bar{y}=\lambda(x) y, \quad \xi=\xi(x),
$$

upon a covariant of degree $d$ and of weight $w$, is to transform it into $\bar{C}$, where

$$
\bar{C}=\frac{\lambda^{d}}{\left(\xi^{\prime}\right)^{\infty}} C .
$$

LIE's theory shows that all other invariants and covariants may be deduced from these by algebraic and differentiation processes. 


\section{§ 2. Canonical forms.}

Equations (2) show that if we make the transformation

$$
y=e^{-\int p_{1} d x} \bar{y}
$$

the coefficients of the resulting equation for $\bar{y}$ will be

$$
\pi_{1}=0, \quad \pi_{2}=P_{2}, \quad \pi_{3}=P_{3}, \quad \pi_{4}=P_{4}+3 P_{2}^{2} .
$$

We shall say that the equation has been put into the semi-canonical form.

From (10) we see that if $\xi(x)$ be chosen so that

$$
\eta^{\prime}-\frac{1}{2} \eta^{2}=\mu=\frac{6}{5} P_{2},
$$

in the resulting equation $\bar{P}_{2}$ will be zero. Since $P_{2}$ is a seminvariant, any transformation of the form $\bar{y}=\lambda y$ will not disturb the equation $P_{2}=0$, and we may again choose $\lambda$ so as to make the coefficient of $d^{3} \bar{y} / d \xi^{3}$ vanish. It is therefore always possible to reduce the equation to the form

$$
\frac{d^{4} \bar{y}}{d \xi^{4}}+4 \pi_{3} \frac{d \bar{y}}{d \xi}+\pi_{4} \bar{y}=0
$$

which we shall call the LAGUERRE-Forsyth canonical form. This is equivalent to assuming $p_{1}=p_{2}=0$ in the original equation.

If $\theta_{3} \neq 0$, we may transform the independent variable so as to make $\bar{\theta}_{3}=1$. In fact we have for an arbitrary transformation

If, therefore, we put

$$
\bar{\theta}_{3}=\frac{1}{\left(\xi^{\prime}\right)^{3}} \theta_{3}
$$

$$
\xi=\int \sqrt[3]{\theta_{3}} d x,
$$

$\bar{\theta}_{3}$ will be equal to unity. We may again by a transformation of the form $\bar{y}=\lambda y$ make $p_{1}$ vanish. The canonical form whick is characterized by the conditions

$$
p_{1}=0, \quad \theta_{3}=1,
$$

we may properly denote as the HALPHEN canonical form.

In our geometrical discussions only the quantity

$$
\eta=\frac{\xi^{\prime \prime}}{\xi^{\prime}}
$$

not $\xi$ itself will be of any importance. $\lambda$ also is an unimportant factor which has no geometrical significance. Equation (13) shows, therefore, that the reducto the LAGUerRe-Forsyth form can always be accomplished in $\infty^{1}$ essentially different ways. It is important to remark that (13) is an equation of the RICCATr form, so that the cross-ratio of any four solutions is constant. 
The HaLPhen form on the other hand can be obtained in just one way, if it exists at all, i. e., if $\theta_{3} \neq 0$. If $\theta_{3}$ vanishes, $\theta_{4}$ may be reduced to unity unless it also is equal to zero. The case when both $\theta_{3}$ and $\theta_{4}$ vanish, is especially simple. The LAGUERRE-ForSYTH form reduces to

$$
\frac{d^{4} \bar{y}}{d \xi^{4}}=0
$$

If two equations of the form (1) can be transformed into each other by a transformation of the kind here considered, we shall call them equivalent. Clearly, for equivalent equations, the corresponding absolute invariants are equal.

If equation (1) is given, the invariants $\theta_{3}, \theta_{4}, \theta_{3.1}$, etc., are known functions of $x$. Conversely, equations (12) show that if $\theta_{3}, \theta_{4}, \theta_{3.1}$ are given as arbitrary functions of $x$, provided that $\theta_{3} \neq 0, P_{2}, P_{3}$ and $P_{4}$ are determined uniquely. If $\theta_{3}=0$, then $\theta_{3.1}=0$ also, and we must assign a further condition. The function

$$
\theta_{4 \cdot 1}=8 \theta_{4} \theta_{4}^{\prime \prime}-9\left(\theta_{4}^{\prime}\right)^{2}-\frac{80}{3} P_{2} \theta_{4}^{2}
$$

is also an invariant. If $\theta_{3}=0$, and $\theta_{4}, \theta_{4 \cdot 1}$ are given, $P_{2}, P_{3}, P_{4}$ are determined uniquely. If both $\theta_{3}$ and $\theta_{4}$ vanish, all invariants are zero, and the equation may be reduced to the form

$$
\frac{d^{4} \eta}{d \xi^{4}}=0
$$

As we may always assume that $p_{1}=0$, we see that the differential equation (1) is essentially determined when its invariants are given as functions of $x$.

The Lagrange adjoint of (1) is

$$
\begin{aligned}
u^{(4)}-4 p_{1} u^{(3)}+6\left(p_{2}-2 p_{1}^{\prime}\right) u^{\prime \prime}-4\left(p_{3}-3 p_{2}^{\prime}+3 p_{1}^{\prime \prime}\right) u^{\prime} \\
+\left(p_{4}-4 p_{3}^{\prime}+6 p_{2}^{\prime \prime}-4 p_{1}^{(3)}\right) u=0 .
\end{aligned}
$$

If $y_{1}, \cdots, y_{4}$ constitute a fundamental system of $(1)$, the minors of $x_{1}, \ldots, x_{4}$ in the determinant

$$
\left|\begin{array}{llll}
x_{1} & x_{2} & x_{3} & x_{4} \\
y_{1} & y_{2} & y_{3} & y_{4} \\
y_{1}^{\prime} & y_{2}^{\prime} & y_{3}^{\prime} & y_{4}^{\prime} \\
y_{1}^{\prime \prime} & y_{2}^{\prime \prime} & y_{3}^{\prime \prime} & y_{4}^{\prime \prime}
\end{array}\right|
$$

multiplied by a common factor, which does not interest us, form a fundamental system of (16). 
If we denote the seminvariants of $(16)$ by $\Pi_{2}, \Pi_{3}, \Pi_{4}$, we have

$$
\Pi_{2}=P_{2}, \quad \Pi_{3}=-P_{3}+3 P_{2}^{\prime}, \quad \Pi_{4}=P_{4}-4 P_{3}^{\prime}+6 P_{2}^{\prime \prime},
$$

whence follows reciprocally

$$
P_{2}=\Pi_{2}, \quad P_{3}=-\Pi_{3}+3 \Pi_{2}^{\prime}, \quad P_{4}=\Pi_{4}-4 \Pi_{3}^{\prime}+6 \Pi_{2}^{\prime \prime} .
$$

The invariants of (16) differ from the invariants of (1) only in this that the sign of $\theta_{3}$ is changed.

\section{§3. Geometrical interpretation.}

If the functions $y_{1}, \cdots, y_{4}$ constitute a fundamental system of (1) we may interpret them as the homogeneous coördinates of a point $P_{y}$ of a curve $C_{y}$ in ordinary space. The coefficients $p_{1}, p_{2}, p_{3}, p_{4}$ of $(1)$ are invariants of the general projective group. The transformation $y=\lambda \bar{y}$ does not change the ratios $y_{1}: y_{2}: y_{3}: y_{4}$, and therefore leaves the curve $C_{y}$ invariant. The transformation $\xi=\xi(x)$ merely changes the parameter in terms of which the coördinates are expressed. It is clear therefore that any system of equations invariant under these transformations expresses a projective property of the curve $C_{y}$ in the vicinity of the point $P$.

The LAGRANGE adjoint of (1) may be taken to represent the same curve in tangential coördinates, or else a reciprocal curve in point coördinates.

We may therefore state the results of $\S 2$ as follows: If the invariants of a curve are given as functions of $x$, the curve is determined except for projective transformations. If the invariants of two curves, except those of weight three, are respectively equal to each other, while the invariants of weight three differ only in sign, the two curves are dualistic to each other. Those curves are self-dual for which $\theta_{3}=0$.

Moreover these latter curves are the only curves which are self-dual in the restricted sense that a dualistic transformation exists which converts every point of the curve into the tangent plane of that point, and vice versâ, while every tangent is converted into itself.

If we put $y=y_{k}(k=1,2,3,4)$ into the expressions for $z, \rho, \sigma$ we obtain three other points $P_{z}, P_{\rho}, P_{\sigma}$, which describe curves $C_{z}, C_{\rho}, C_{\sigma}$ as $x$ varies, curves which are closely connected with $C_{y} \cdot P_{z}$ is clearly a point on the tangent of $C_{y}$ constructed at $P_{y} ; P_{\rho}$ is in the plane osculating $C_{y}$ at $P_{y}$, while $\boldsymbol{P}_{\sigma}$ is outside of this plane. These four points are never coplanar except at those exceptional points of $C_{y}$ whose osculating planes are stationary, i. e., have more than three consecutive points in common with the curve.

In order to study the curve $C_{y}$ in the vicinity of $P_{y}$, it will therefore be convenient to introduce the tetrahedron $P_{y} P_{z} P_{\rho} P_{\sigma}$ as tetrahedron of reference, with the further convention that if any expressions of the form

$$
u_{k}=\alpha_{1} y_{k}+\alpha_{2} z_{k}+\alpha_{3} \rho_{k}+\alpha_{4} \sigma_{k}
$$


offer themselves the cöordinates of the corresponding point $P_{u}$ shall be

$$
\left(\alpha_{1}, \alpha_{2}, \alpha_{3}, \alpha_{4}\right) \text {. }
$$

In writing $u_{k}$ the index $k$ may be suppressed, so that a single expression

$$
\alpha_{1} y+\alpha_{2} z+\alpha_{3} \rho+\alpha_{4} \sigma
$$

represents the point $\left(\alpha_{1}, \alpha_{2}, \alpha_{3}, \alpha_{4}\right)$ in this special system of coördinates.

As the independent variable $x$ is changed, the tetrahedron of reference is changed in accordance with equations (9). $P_{y}$ of course remains the same; $P_{z}$ is changed into $P_{\bar{z}}$, which may obviously be any point on the tangent: etc. Thus while an arbitrary transformation of the parameter $x$ does not affect the curve $C_{y}$ itself, it does very materially affect the semi-covariant curves $C_{z}, C_{\rho}$ and $C_{\sigma}$. It is clear however that two transformations $\xi=\xi(x)$, for which $\eta=\xi^{\prime \prime} / \xi^{\prime}$ has the same value, are geometrically equivalent. We may also, without affecting the position of the points $P_{z}, P_{\rho}, P_{\sigma}$, assume that (1) is written in the semi-canonical form, so that $p_{1}=0$. For, in order to put (1) into the semi-canonical form, we need only multiply $y$ by a certain factors $\lambda$, which will then also appear multiplied into the semi-covariants $z, \rho$ and $\sigma$.

Let us then assume $p_{1}=0$. We shall have $z=y^{\prime}$. If we differentiate (1) and eliminate $y$ between the resulting equation and (1), we shall find

$$
\begin{aligned}
& \left(P_{4}+3 P_{2}^{2}\right) z^{(4)}-\left(P_{4}^{\prime}+6 P_{2} P_{2}^{\prime}\right) z^{(3)}+6 P_{2}\left(P_{4}+3 P_{2}^{2}\right) z^{\prime \prime} \\
& \quad+\left[\left(6 P_{2}^{\prime}+4 P_{3}\right)\left(P_{4}+3 P_{2}^{2}\right)-6 P_{2}\left(P_{4}^{\prime}+6 P_{2} P_{2}^{\prime}\right)\right] z^{\prime} \\
& \quad+\left[\left(4 P_{3}^{\prime}+P_{4}+3 P_{2}^{2}\right)\left(P_{4}+3 P_{2}^{\prime 2}\right)-4 P_{3}\left(P_{4}^{\prime}+6 P_{2} P_{2}^{\prime}\right)\right] z=0,
\end{aligned}
$$

if $P_{4}+3 P_{2}^{2} \neq 0$. If $P_{4}+3 P_{2}^{2}=0$ we find

$$
z^{(3)}+6 P_{2} z^{\prime}+4 P_{3} z=0 .
$$

Equation (18) determines the curve $C_{z}$ in the same way as (1) determines $C_{y}$. But if $P_{4}+3 P_{z}^{2}=0, z$ satisfies (19) showing that the curve $C_{z}$ is in this case a plane curve. Therefore, if the variable $\xi$ be so chosen as to make $P_{4}+3 P_{2}^{2}=0$, the corresponding curve $C_{\bar{z}}$ is a plane section of the developable surface whose cuspidal edge is $C_{y}$. In harmony with this, equations (10) show that the most general value of $\eta$, which satisfies the condition $P_{4}+3 P_{2}^{2}=0$, contains three arbitrary constants, as it should since there are $\infty^{3}$ planes in space.

We shall need to consider the ruled surfaces generated by those edges of our tetrahedron which meet in $P_{y}$. (Of these we know one immediately, namely the developable which has $C_{y}$ as its edge of regression, and of which $P_{y} P_{z}$ is a generator. The ruled surface generated by $P_{y} P_{\rho}$ clearly has $C_{y}$ as an asymptotic curve; for, the plane $P_{y} P_{\rho} P_{z}$ is both osculating plane of $C_{y}$ at $P_{y}$, and 
tangent plane of the surface at $P_{y}$. If we assume $p_{1}=0$, this ruled surface may be studied by means of the equations

$$
y^{\prime \prime}+P_{2} y-\rho=0,
$$

$$
\rho^{\prime \prime}+\left(4 P_{3}-2 P_{2}^{\prime}\right) y^{\prime}+\left(P_{4}-P_{2}^{\prime \prime}-2 P_{2}^{2}\right) y+5 P_{2} \rho=0,
$$

in accordance with the general theory of ruled surfaces as developed in former papers of the author. To prove (20) we need only differentiate twice the expression for $\rho$, express $y^{(3)}$ and $y^{(t)}$ in terms of $y, z, \rho, \sigma$, and eliminate $z$ and $\sigma$.

The ruled surface generated by $P_{y} P_{\sigma}$ is especially important. We have,

$$
\sigma=y^{(3)}+3 p_{1} y^{(2)}+3 p_{2} y^{\prime}+p_{3} y,
$$

whence

$$
\sigma^{\prime}=y^{(4)}+3 p_{1} y^{(3)}+\left(3 p_{1}^{\prime}+3 p_{2}\right) y^{\prime \prime}+\left(3 p_{2}^{\prime}+p_{3}\right) y^{\prime}+p_{3}^{\prime} y,
$$

From (21) we find

$$
\begin{aligned}
& \sigma^{\prime \prime}=y^{(5)}+3 p_{1} y^{(4)}+3\left(2 p_{1}^{\prime}+p_{2}\right) y^{(3)} \\
& +\left(3 p_{1}^{\prime \prime}+6 p_{2}^{\prime}+p_{3}\right) y^{\prime \prime}+\left(3 p_{2}^{\prime \prime}+2 p_{3}^{\prime}\right) y^{\prime}+p_{3}^{\prime \prime} y .
\end{aligned}
$$

$$
y^{(3)}=\sigma-3 p_{1} y^{\prime \prime}-3 p_{2} y^{\prime}-p_{3} y,
$$

$$
\begin{aligned}
y^{(4)}=\sigma^{\prime}-3 p_{1} \sigma-3\left(p_{1}^{\prime}+\right. & \left.p_{2}-3 p_{1}^{2}\right) y^{\prime \prime} \\
& \quad-\left(3 p_{2}^{\prime}+p_{3}-9 p_{1} p_{2}\right) y^{\prime}-\left(p_{3}^{\prime}-3 p_{1} p_{3}\right) y .
\end{aligned}
$$

If we substitute these values in (1) we obtain the equation

$$
3\left(p_{2}-p_{1}^{\prime}-p_{1}^{2}\right) y^{\prime \prime}+3\left(p_{3}-p_{2}^{\prime}-p_{1} p_{2}\right) y^{\prime}+\sigma^{\prime}
$$

$$
+\left(p_{4}-p_{1} p_{3}-p_{3}^{\prime}\right) y+p_{1} \sigma=0,
$$

where the coefficient of $y^{\prime \prime}$ is $3 P_{2}$.

Let us differentiate both members of this equation, and eliminate $y^{\prime \prime}$ and $y^{(3)}$ by means of (23) and (24). We shall find

$$
\begin{aligned}
3 P_{2} \sigma^{\prime \prime}=\left(q_{3} r_{3}+3 P_{2} q_{4}\right) y^{\prime}+ & \left(3 P_{2} q_{1}-q_{3}\right) \sigma^{\prime} \\
& +\left(r_{4} q_{3}+3 P_{2} q_{5}\right) y+\left(3 P_{2} q_{2}-p_{1} q_{3}\right) \sigma,
\end{aligned}
$$

where

$$
\begin{aligned}
& q_{1}=-p_{1}, \quad q_{2}=2 p_{1}^{\prime}-3 p_{2}+3 p_{1}^{2}, \quad q_{3}=-3 P_{3}+3 p_{1} P_{2}, \\
& q_{4}=-2 p_{3}^{\prime}+3 p_{2}^{\prime \prime}+3 p_{1} p_{2}^{\prime}-6 p_{1}^{\prime} p_{2}-p_{4}+p_{1} p_{3}+9 p_{2}^{2}-9 p_{1}^{2} p_{2}, \\
& q_{5}=-\left(p_{4}^{\prime}-p_{3}^{\prime \prime}-p_{1} p_{3}^{\prime}-p_{1}^{\prime} p_{3}\right)+3 p_{3}\left(p_{2}-p_{1}^{\prime}-p_{1}^{2}\right), \\
& r_{3}=-3\left(p_{3}-p_{2}^{\prime}-p_{1} p_{2}\right), \\
& r_{4}=-\left(p_{4}-p_{3}^{\prime}-p_{1} p_{3}\right) .
\end{aligned}
$$


Equations (24) and (25) define the ruled surface generated by $P_{y} P_{\sigma}$. If we assume $p_{1}=0$, we find

$$
\begin{aligned}
& y^{\prime \prime}+p_{11} y^{\prime}+p_{12} \sigma^{\prime}+q_{11} y+q_{12} \sigma=0 \\
& \sigma^{\prime \prime}+p_{21} y^{\prime}+p_{22} \sigma^{\prime}+q_{21} y+q_{22} \sigma=0
\end{aligned}
$$

where

$$
\begin{aligned}
& p_{11}=\frac{P_{3}-P_{2}^{\prime}}{P_{2}}, \quad p_{12}=\frac{1}{3 P_{2}}, \quad q_{11}=\frac{P_{4}+3 P_{2}^{2}-P_{3}^{\prime}}{3 P_{2}}, \quad q_{12}=0, \\
& p_{21}=\frac{1}{P_{2}^{-}}\left[-3 P_{3}^{2}+3 P_{3} P_{2}^{\prime}+2 P_{2} P_{3}^{\prime}-3 P_{2} P_{2}^{\prime \prime}+P_{2} P_{4}-6 P_{2}^{3}\right], \\
& p_{22}=-\frac{P_{3}}{P_{2}} \\
& q_{21}=\frac{1}{P_{2}}\left[-P_{3} P_{4}-6 P_{2}^{2} P_{3}+P_{3} P_{3}^{\prime}+P_{2} P_{4}^{\prime}+6 P_{2}^{2} P_{2}^{\prime}-P_{2} P_{3}^{\prime \prime}\right], \\
& q_{22}=3 P_{2} .
\end{aligned}
$$

If (1) is written in the LAGUERRE-Forsyth form, $P_{2}=0$. In that case the two equations $(27)$ reduce to the single equation

$$
P_{3} y^{\prime}+\frac{1}{3} \sigma^{\prime}+\frac{1}{8}\left(P_{4}-P_{3}^{\prime}\right) y=0,
$$

which proves that in this case the surface generated by $P_{y} P_{\sigma}$ is developable. For in this case the tangents constructed respectively to $C_{y}$ at $P_{y}$ and to $C_{\sigma}$ at $P_{\sigma}$ are coplanar. Moreover, only if $P_{2}=0$ will the surface generated by $P_{y} P_{\sigma}$ be a developable.

Let

$$
\tau=\lambda y+\mu \sigma
$$

represent the point at which $P_{y} P_{\sigma}$ intersects the edge of regression of the developable. Then, since $P_{y} P_{\sigma}$ must be tangent to the edge of regression, we shall have $\tau^{\prime}=\alpha y+\beta \sigma$, or

$$
\left(\lambda^{\prime}-\alpha\right) y+\left(\mu^{\prime}-\beta\right) \sigma+\lambda y^{\prime}+\mu \sigma^{\prime}=0 .
$$

But according to (29)

so that

$$
\sigma^{\prime}=-\left(P_{1}-P_{3}^{\prime}\right) y-3 P_{3} y^{\prime}
$$

$$
\left(\lambda^{\prime}-\alpha\right) y+\left(\mu^{\prime}-\beta\right) \sigma+\lambda y^{\prime}-\mu\left[\left(P_{4}-P_{3}^{\prime}\right) y+3 P_{3} y^{\prime}\right]=0,
$$

where for $y^{\prime}$ we could also write $z$. Such a relation between $P_{y}, P_{z}, P_{\sigma}$ would, however, make these three points collinear, and, therefore, $P_{y}, P_{z}, P_{\rho}, P_{\sigma}$ coplanar, unless all of the coefficients are zero. We have seen, however, that this 
can happen only for such points $P_{y}$ at which the osculating plane is stationary. We must have, therefore,

$$
\lambda^{\prime}-\alpha-\mu\left(P_{4}-P_{3}^{\prime}\right)=0, \quad \mu^{\prime}-\not \beta=0, \quad \lambda-3 \mu P_{3}=0,
$$

whence

$$
\lambda=3 \mu P_{3}, \quad \beta=\mu^{\prime}, \quad \alpha=\lambda^{\prime}-\mu\left(P_{4}-P_{3}^{\prime}\right) .
$$

We see, therefore, that

$$
\tau=3 P_{3} y+\sigma
$$

represents the edge of regression of the developable to which the ruled surface generated by $P_{y} P_{\sigma}$ reduces when $P_{2}=0$.

If $p_{1}=0$ and $P_{2}=0$, equations (2) and (10) show that the most general transformations of the variables, which do not disturb these conditions, satisfy the equations

$$
\frac{\lambda^{\prime}}{\lambda}+\frac{3}{2} \frac{\xi^{\prime \prime}}{\xi^{\prime}}=0, \quad \mu=\eta^{\prime}-\frac{1}{2} \eta^{2}=0,
$$

which give on integration

$$
\lambda=\frac{C}{\left(\xi^{\prime}\right)^{i}}, \quad \eta=\frac{-2 c}{1+c x} .
$$

If we transform $\tau$ under this assumption, we find that it is converted into

$$
\bar{\tau}=\frac{1}{\left(\xi^{\prime}\right)^{3} \lambda}\left[\sigma+\frac{8}{2} \eta \rho+\frac{8}{2} \eta^{2} z+\left(\frac{8}{4} \eta^{3}+3 P_{3}\right) y\right],
$$

where $\eta$ may have any numerical value.

Let us recapitulate. The ruled surfaces generated by $P_{y} P_{\bar{\sigma}}$ are infinite in number. Their general expression involves an arbitrary function $\eta$. Among these surfaces there exists a single infinity of developables. If $P_{2}=0$, the surface generated by $P_{y} P_{\sigma}$ is one of these, and the locus of $P_{\tau}$ is its edge of regression, where

$$
\tau=3 P_{3} y+\sigma,
$$

$P_{\tau}$ being the point where $P_{y} P_{\sigma}$ intersects the edge of regression. If we construct all the $\infty^{1}$ lines $P_{y} P_{\bar{\sigma}}$ through $P_{y}$, which are generators of the above mentioned family of developables, and mark upon each of them the point $\boldsymbol{P}_{\bar{\tau}}$ where it intersects the cuspidal edge of the developable to which it belongs, the locus of these points is a twisted cubic curve. The equations of this curve referred to a parameter $\eta$ and to the fundamental tetrahedron $P_{y} P_{z} P_{p} P_{\sigma}$ are

$$
x_{1}=3 P_{3}+\frac{8}{4} \eta^{3}, \quad x_{2}=\frac{8}{2} \eta^{2}, \quad x_{3}=\frac{3}{2} \eta, \quad x_{4}=1 .
$$

We shall see later that this cubic has five consecutive points in common with the curve $C_{y}$ at $P_{y}$, i. e., that it has at this point with $C_{y}$ a contact of the fourth 
order. We shall speak of it as the torsal cubic of $P_{y}$, on account of its connection with the developables which we have just been considering.

Equations (31) give the parametric equations of the torsal cubic referred to a special tetrahedron of reference for which $P_{2}=0$. We shall need its equations in a more general form. These may be easily obtained. Consider the expression

$$
\lambda=\left(3 \theta_{3}+\frac{3}{10} P_{2}^{\prime}+\frac{6}{5} P_{2} t+\frac{3}{4} t^{3}\right) y+\left(\frac{6}{5} P_{2}+\frac{3}{2} t^{2}\right) z+\frac{3}{2} t \rho+\sigma
$$

in which $t$ may for the moment be regarded as a parameter independent of $x$. Denote by $\bar{\lambda}$ the corresponding expression formed from the quantities $\bar{P}_{2}, \bar{P}_{3}$, etc., $\bar{y}, \bar{z}, \bar{\rho}, \bar{\alpha}$ after the general transformation $\xi=\xi(x)$. We shall find that $\left(\xi^{\prime}\right)^{3} \bar{\lambda}$. is equal to $\lambda$ after $t$ has been replaced by $t \xi^{\prime}+\eta=t_{1}$. But of course this transformation may be chosen so as to make $\bar{P}_{2}=0$, which would make $\bar{\lambda}$ identical with $\bar{\tau}$ except for the notation.

We see, therefore, that the expression $\lambda$, or the equations

$$
\begin{aligned}
& x_{1}=3 \theta_{3}+\frac{3}{1} \delta P_{2}^{\prime}+\frac{6}{5} P_{2} \eta+\frac{3}{4} \eta^{3}, \\
& x_{2}=\frac{6}{5} P_{2}+\frac{3}{2} \eta^{2}, \quad x_{3}=\frac{3}{2} \eta, \quad x_{4}=1,
\end{aligned}
$$

represent the torsal cubic referred to the fundamental tetrahedron $P_{y} P_{z} P_{\rho} P_{\sigma}$ when this is chosen in as general a way as is compatible with its definition.

If in (32) $t$ is chosen as a function of $x$, as $x$ varies we obtain a curve on the surface formed by the totality of torsal cubics. If in particular $t$ satisfies as function of $x$ the differential equation

$$
t^{\prime}-\frac{1}{2} t^{2}=\frac{6}{5} P_{2},
$$

we obtain the cuspidal edge of one of the developables.

\section{§. The osculating cubic, conic and linear complex.}

A space cubic is determined by six of its points provided that no four of these points are coplanar. If, therefore, we take upon $C_{y}$, besides $P_{y}$, five other points, we shall in general obtain a perfectly definite space cubic determined by these six points. As these points approach coincidence with $P_{y}$, this cubic will in general approach a limit, which shall be called the osculating cubic. We proceed to find its equations.

Let $P_{y}$ correspond to the value of $x=a$, which we shall suppose is an ordindry point for our differential equation. Then $y$ may be developed by Taylor's theorem into a series proceeding according to powers of $x-a$. By putting $x-a=x^{\prime}$ the development will be in powers of $x^{\prime}$. We may therefore assume in the first place that $a=0$. Let us assume, further, that $p_{1}=0$ and $P_{2}=0$. Then we shall have from (5) and (6), 


$$
\begin{aligned}
& y^{\prime}=z, \quad y^{\prime \prime}=\rho, \quad y^{(3)}=-P_{3} y+\sigma, \quad y^{(4)}=-P_{4} y-4 P_{3} z, \\
& y^{(5)}=-P_{4}^{\prime} y-\left(P_{4}+4 P_{3}^{\prime}\right) z-4 P_{3} \rho .
\end{aligned}
$$

In accordance with the definition of our coördinates, we denote the coefficients of $y, z, \rho, \sigma$ in this expansion carried as far as $x^{5}$, by $y_{1}, y_{2}, y_{3}, y_{4}$. 'We may of course multiply these quantities by a common factor since the coördinates are homogeneous. We shall multiply by 120 so as to clear of fractions. This gives

$$
\begin{aligned}
& y_{1}=120-20 P_{3} x^{3}-5 P_{4} x^{4}-P_{4}^{\prime} x^{5}+\cdots \\
& y_{2}=120 x-20 P_{3} x^{4}-\left(4 P_{3}^{\prime}+P_{4}\right) x^{5}+\cdots, \\
& y_{3}=60 x^{2}-4 P_{3} x^{5}+\cdots, \\
& y_{4}=20 x^{3}+\cdots
\end{aligned}
$$

We see at once that the following equations are exact up to terms no higher than the fifth order:

$$
3 y_{2} y_{4}-2 y_{3}^{2}=0, \quad 5\left(2 y_{1} y_{3}-y_{2}^{2}\right)-6 P_{3} y_{3} y_{4}=0 \text {. }
$$

These equations must be satisfied by the coördinates of any point of the osculating cubic, since this must have contact of the fifth order with $C_{y}$ at $P_{y}$. They are therefore its equations, referred to this special tetrahedron of reference. In terms of a parameter $t$ we may write

$$
x_{1}=15+12 P_{3} t^{3}, \quad x_{2}=30 t, \quad x_{3}=30 t^{2}, \quad x_{4}=20 t^{3} .
$$

The equation

$$
3 x_{2} x_{4}-2 x_{3}^{2}=0
$$

is that of a cone whose vertex is $P_{y}$ and which contains the osculating cubic. It may also be obtained by determining that cone of the second order with its vertex at $P_{y}$ which has the closest possible contact with $C_{y}$, viz. contact of the fifth order. We shall speak of it as the osculating cone. We notice at once that the torsal cubic also lies upon the osculating cone. This is shown by equations (31) which are referred to the same system of coördinates as that employed here. If we put $\eta=1 / t$ in (31) and if we multiply by $20 t^{3},(31)$ becomes

$$
x_{1}=15+60 P_{3} t^{3}, \quad x_{2}=30 t, \quad x_{3}=30 t^{2}, \quad x_{4}=20 t^{3},
$$

which differs from (37) only in having $5 P_{3}$ in place of $P_{3}$.

By a method of reasoning precisely similar to that of the last paragraph, we find that the expression

$$
\left(12 P_{3}-12 P_{2}^{\prime}+24 P_{2} \tau+15 \tau^{3}\right) y+20\left(\frac{6}{6} P_{2}+\frac{3}{2} \tau^{2}\right) z+30 \tau \rho+20 \sigma
$$


represents an arbitrary point of the osculating cubic when the tetrahedron of reference is not restricted to the condition $P_{2}=0$. For this expression remains invariant under the general transformation $\xi=\xi(x)$, and reduces to (37) for $P_{2}=0$, if $\tau=1 / t$.

The equation of the plane, which osculates the osculating cubic at the point whose parameter is $\tau$, turns out to be

where

$$
\dot{u}_{1} x_{1}+u_{2} x_{2}+u_{3} x_{3}+u_{4} x_{4}=0,
$$

$$
\begin{aligned}
& u_{1}=-20, \quad u_{2}=30 \tau, \quad u_{3}=16 P_{2}-30 \tau^{2}, \\
& u_{4}=12 P_{3}-12 P_{2}^{\prime}-36 P_{2} \tau+15 \tau^{3} .
\end{aligned}
$$

For every value of $\tau$ this intersacts the osculating plane, $x_{4}=0$, in a straight line

$$
-20 x_{1}+30 \tau x_{2}+\left(16 P_{2}-30 \tau^{2}\right) x_{3}=0 .
$$

The envelope of these lines will be obtained by eliminating $\tau$ between this equation and that obtained from it by partial differentiation with respect to $\tau$; the latter equation is

We thus find

$$
30 x_{2}-60 \tau x_{3}=0 \text {. }
$$

$$
-40 x_{1} x_{3}+15 x_{2}^{2}+32 P_{2} x_{3}^{2}=0 \text {, }
$$

the equation of the osculating conic, which may be defined as a part of the intersection of the developable of the osculating cubic with the osculating plane. The other part of this intersection is the tangent, which must be counted twice.

It is not without interest to verify that (37) represents the osculating cubic by another method. We have from (35) as non-homogeneous coördinates of the points of $C_{y}$ in the vicinity of $P_{y}$

$$
\begin{gathered}
\frac{y_{2}}{y_{1}}=x-{ }_{1} \frac{1}{2} \delta\left(P_{3}^{\prime}-4 P_{4}\right) x^{5}+\cdots, \quad \frac{y_{3}}{y_{1}}=\frac{1}{2} x^{2}+\frac{1}{20} P_{3} x^{5}+\cdots, \\
\frac{y_{4}}{y_{1}}=\frac{1}{6} x^{3}+\cdots .
\end{gathered}
$$

From (37) we find for the points of the osculating cubic

$$
\frac{x_{2}}{x_{1}}=2 t-\frac{8}{6} P_{3} t^{4}+\cdots, \quad \frac{x_{3}}{x_{1}}=2 t^{2}-\frac{8}{5} P_{3} t^{5}+\cdots, \quad \frac{x_{4}}{x_{1}}=\frac{4}{3} t^{3}+\cdots
$$

If we put

$$
t=\frac{1}{2} x\left[1+\frac{1}{10} P_{3} x^{3}-\frac{1}{80}\left(P_{3}^{\prime}+P_{4}\right) x^{4}+\cdots\right],
$$

these two expansions coincide up to terms of the fifth order. For the torsal cubic we have according to (31a),

$$
\frac{x_{2}}{x_{1}}=2 t-8 P_{3} t^{4} \cdots, \quad \frac{x_{3}}{x_{1}}=2 t^{2}-8 P_{3} t^{5}+\cdots, \quad \frac{x_{4}}{x_{1}}=\frac{4}{8} t^{3}+\cdots
$$


If we put into these equations

$$
t=\frac{1}{2} x\left(1+a x+b x^{2}+c x^{3}+d x^{4}+\cdots\right),
$$

we find that these expansions will agree with (41) up to terms of the fourth order for

$$
a=0, \quad b=0, \quad c=\frac{1}{2} P_{3} .
$$

but that it is impossible to make them agree with (41) any farther unless $P_{3}=0$. In general, therefore, the torsal cubic has with $C_{y} a$ contact of the fourth order. Only if $\theta_{3}=0$ may the order of contact be higher. In that case the torsal and osculating cubics coincide.

We proceed to deduce the equation of the osculating linear complex, i. e., of that linear complex determined by five consecutive tangents of the curve. We assume again $p_{1}=0$ and $P_{2}=0$. Denote by $Y$ and $Z$ the expansions of $y$ and $z$ in the vicinity of $P_{y}$. Then we have up to terms of the fourth order

$$
\begin{aligned}
& Y=y\left(1-\frac{1}{6} P_{3} x^{3}-\frac{1}{24} P_{4} x^{4}\right)+z\left(x-\frac{1}{6} P_{3} x^{4}\right)+\frac{1}{2} \rho x^{2}+\frac{1}{6} \rho x^{3}, \\
& \begin{array}{r}
Z=y\left(-\frac{1}{2} P_{3} x^{2}-\frac{1}{6} P_{4} x^{3}-\frac{1}{24} P_{4}^{\prime} x^{4}\right)+z\left[1-\frac{2}{3} P_{3} x^{3}-\frac{1}{24}\left(4 P_{3}^{\prime}+P_{4}\right) x^{4}\right] \\
+\rho\left(x-\frac{1}{6} P_{3} x^{4}\right)+\sigma \frac{1}{2} x^{2} .
\end{array}
\end{aligned}
$$

If we denote the coefficients of $y, z, \rho, \sigma$ in these two expressions by $y_{1}, \cdots, y_{4}$ and $z_{1}, \ldots, z_{4}$, respectively, the Plückerian line-cöordi nates of the tangent will be

whence

$$
\omega_{i k}=y_{i} z_{k}-y_{k} z_{i}
$$

$$
\begin{array}{lll}
\omega_{12}=1-\frac{1}{6} P_{8} x^{3}+\frac{1}{8} P_{4} x^{4}+\cdots, & \omega_{13}=x-\frac{1}{12} P_{3} x^{4}+\cdots, & \omega_{14}=\frac{1}{2} x^{2}+\cdots, \\
\omega_{23}=\frac{1}{2} x^{2}+\cdots, & \omega_{24}=\frac{1}{3} x^{3}+\cdots, & \omega_{34}=\frac{1}{12} x^{4}+\cdots,
\end{array}
$$

Therefore, the equation of the osculating linear complex, referred to the special tetrahedron of reference, is

$$
\omega_{14}-\omega_{23}=0 \text {. }
$$

We might have obtained this complex in another way. For, it is clear that the null-system of the osculating cubic will be the same as that determined by the osculating linear complex. We shall, instead, set up the null-system of the torsal cubic in its general form. We shall see that the linear complex determined by the torsal cubic coincides with the osculating linear complex.

We have the equations of the torsal cubic

$$
\begin{aligned}
& x_{1}=60 \theta_{3}+6 P_{2}^{\prime}+24 P_{2} \eta+15 \eta^{3}, \\
& x_{2}=24 P_{2}+30 \eta^{2}, \quad x_{3}=30 \eta, \quad x_{4}=20 .
\end{aligned}
$$


The coördinates of the plane, which osculates the torsal cubic at the point whose parameter is $\eta$, are

$$
\begin{aligned}
& u_{1}=-180, \quad u_{2}=270 \eta, \quad u_{3}=144 P_{2}-270 \eta^{2}, \\
& u_{4}=540 \theta_{3}+54 P_{2}^{\prime}-324 P_{2} \eta+135 \eta^{3} .
\end{aligned}
$$

If we put in (43) $\eta=\eta_{k}(k=1,2,3)$ we obtain three points on the cubic. The coördinates of their plane must be prcportional to

$$
\begin{aligned}
& v_{1}=-180, \quad v_{2}=90\left(\eta_{1}+\eta_{2}+\eta_{3}\right), \\
& v_{3}=144 P_{2}-90\left(\eta_{2} \eta_{3}+\eta_{3} \eta_{1}+\eta_{1} \eta_{2}\right), \\
& v_{4}=540 \theta_{3}+54 P_{2}^{\prime}-108 P_{2}\left(\eta_{1}+\eta_{2}+\eta_{3}\right)+135 \eta_{1} \eta_{2} \eta_{3},
\end{aligned}
$$

for each of these expressions must be a symmetric function of $\eta_{1}, \eta_{2}, \eta_{3}$ of order not higher than the third, and for $\eta_{1}=\eta_{2}=\eta_{3}=\eta$ we must have $v_{k}$ proportional to $u_{k}$. Similarly, the point in which the three osculating planes at $\eta_{1}, \eta_{2}, \eta_{3}$ intersect, must have its cöordinates proportional to

$$
\begin{aligned}
& \omega x_{1}=60 \theta_{3}+6 P_{2}^{\prime}+8 P_{2}\left(\eta_{1}+\eta_{2}+\eta_{3}\right)+15 \eta_{1} \eta_{2} \eta_{3}, \\
& \omega x_{2}=24 P_{2}+10\left(\eta_{2} \eta_{3}+\eta_{3} \eta_{1}+\eta_{1} \eta_{2}\right), \\
& \omega x_{3}=10\left(\eta_{1}+\eta_{2}+\eta_{3}\right) ; \quad \omega x_{4}=20 .
\end{aligned}
$$

If we eliminate $\eta_{1}, \eta_{2}, \eta_{3}$ between (45) and (46) and change slightly the factor of proportionality, we find

or

$$
\begin{array}{ll}
\omega v_{1}=-x_{4}, & \omega v_{3}=-x_{2}+2 P_{2} x_{4}, \\
\omega v_{2}=+x_{3}, & \omega v_{4}=+x_{1}-2 P_{2} x_{3},
\end{array}
$$

$$
\begin{array}{ll}
\omega^{\prime} x_{1}=+2 P_{2} v_{2}+v_{4}, & \omega^{\prime} x_{3}=+v_{2}, \\
\omega^{\prime} x_{2}=-2 P_{2} v_{1}-v_{3}, & \omega^{\prime} x_{4}=-v_{1},
\end{array}
$$

as the equations of the null-system defined by the torsal cubic.

A point $y_{1}, y_{2}, y_{3}, y_{4}$ lies in the plane corresponding to $x_{1}, x_{2}, x_{3}, x_{4}$ if

$$
\sum_{k=1}^{4} v_{k} y_{k}=0 \text {. }
$$

Therefore, the lines which pass through the point $x_{1}, x_{2}, x_{3}, x_{4}$ and lie in the plane corresponding to it in the null-system, satisfy the equation

$$
\omega_{14}-2 P_{2} \omega_{34}-\omega_{23}=0 \text {. }
$$

If the tetrahedron of reference be so chosen as to make $P_{2}=0$, this equation is identical with (42). Therefore (48) represents the osculating linear complex 
when the tetrahedron of reference is general. The osculating and torsal cubics are curves of this complex.

If $P_{2}$ is finite the complex (48) is not special. We see therefore that only those values of $x$ for which $P_{2}=\infty$ can give points of the curve at which five consecutive tangents have a straight line intersector.

Let us proceed to deduce the equation of the osculating linear complex belonging to a point of $C_{y}$ infinitesimally close to $P_{y}$. If we change $x$ by an infinitesimal amount $\delta x$, we find for the coördinates of the vertices of the new tetrahedron of reference

$\bar{y}=y+y^{\prime} \delta x=\left(1-p_{1} \delta x\right) y+z \delta x$,

$\bar{z}=z+z^{\prime} \delta x=-P_{2} \delta x \cdot y+\left(1-p_{1} \delta x\right) z+\rho \delta x$,

$\bar{\rho}=\rho+\rho^{\prime} \delta x=-\left(P_{3}-P_{2}^{\prime}\right) \delta x \cdot y-2 P_{2} \delta x \cdot z+\left(1-p_{1} \delta x\right) \rho+\sigma \delta x$,

$\bar{\sigma}=\sigma+\sigma^{\prime} \delta x=-\left(P_{4}-P_{3}^{\prime}\right) \delta x \cdot y-3\left(P_{3}-P_{2}^{\prime}\right) \delta x \cdot z$

$$
-3 P_{2} \delta x \cdot \rho+\left(1-p_{1} \delta x\right) \sigma \text {. }
$$

Therefore, if a point has the coördinates $\overline{\bar{x}}_{1}, \ldots, \bar{x}_{4}$ in the new system of coördinates, and $x_{1}, \cdots, x_{4}$ in the old, we shall have

$$
x_{1}=\left(1-p_{1} \delta x\right) \bar{x}_{1}-P_{2} \delta x \cdot \bar{x}_{2}-\left(P_{3}-P_{2}^{\prime}\right) \delta x \cdot \bar{x}_{3}-\left(P_{4}-P_{3}^{\prime}\right) \delta x \cdot \bar{x}_{1},
$$

Therefore the infinitesimal changes in the coördinates in the sense new minus old, will be

$$
\begin{aligned}
& \delta x_{1}=\left[p_{1} x_{1}+P_{2} x_{2}+\left(P_{3}-P_{2}^{\prime}\right) x_{3}+\left(P_{4}-P_{3}^{\prime}\right) x_{4}\right] \delta x, \\
& \delta x_{2}=\left[-x_{1}+p_{1} x_{2}+2 P_{2} x_{3}+3\left(P_{3}-P_{2}^{\prime}\right) x_{4}\right] \delta x, \\
& \delta x_{3}=\left(-x_{2}+p_{1} x_{3}+3 P_{2} x_{4}\right) \delta x, \\
& \delta x_{4}=\left(-x_{3}+p_{1} x_{4}\right) \delta x .
\end{aligned}
$$

Referred to the new tetrahedron of reference, the equation of the complex osculating $C_{y}$ at the point corresponding to $x+\delta x$ will be

where

$$
\bar{\omega}_{14}-2 \bar{P}_{2} \bar{\omega}_{34}-\bar{\omega}_{23}=0 \text {, }
$$

$$
\begin{aligned}
\bar{P}_{2} & =P_{2}+P_{2}^{\prime} \delta x, & \bar{\omega}_{i x} & =\bar{x}_{i} \bar{y}_{k}-\bar{x}_{k} \bar{y}_{i}, \\
\bar{x}_{i} & =x_{i}+\delta x_{i}, & \bar{y}_{i} & =y_{i}+\delta y_{i},
\end{aligned}
$$

if $\bar{x}_{i}$ and $\bar{y}_{i}$ denote the coördinates of two points on a line of the complex referred to the new tetrahedron of reference. Making the calculations we find

$$
\begin{aligned}
& \bar{\omega}_{14}=\omega_{14}+\left[-\omega_{13}+2 p_{1} \omega_{14}-P_{2} \omega_{42}+\left(P_{3}-P_{2}^{\prime}\right) \omega_{34}\right] \delta x, \\
& \bar{\omega}_{34}=\omega_{34}+\left(2 p_{1} \omega_{34}+\omega_{42}\right) \delta x, \\
& \bar{\omega}_{23}=\omega_{23}+\left[2 p_{1} \omega_{23}-3 P_{2} \omega_{42}-\omega_{13}-3\left(P_{3}-P_{2}^{\prime}\right) \omega_{34}\right] \delta x .
\end{aligned}
$$


If we substitute in (50), we find as the equation of the linear complex osculating $C_{y}$ at a point infinitesimally close to $P_{y}$,

$$
\left(\omega_{14}-2 P_{2} \omega_{34}-\omega_{23}\right)\left(1+2 p_{1} \delta x\right)+4 \theta_{3} \omega_{34} \delta x=0 .
$$

This coincides with the linear complex osculating $C_{y}$ at $P_{y}$, if, and only if, $\theta_{3}=0$. Therefore, if the invariant $\theta_{3}$ vanishes identically, the tangents of the curve $C_{y}$ belong to a linear complex. If it does not vanish identically, those values of $x$ for which it does vanish correspond to points of the curve at which the osculating linear complex hyperosculates the curve.

This result, which has been known a long time, is here derived in a novel manner, which has above all the advantages of clearness. It mav also be obtained by setting up the linear differential equation of the sixth order satisfied by the six line coördinates

$$
\omega_{i k}=y_{i} y_{k}^{\prime}-y_{k} y_{i}^{\prime},
$$

of the tangent, and noting that this reduces to the fifth order if, and only if, $\theta_{3}=0$. This is the method of Halphen.

A former result may now be stated as follows: The osculating and torsal cubics of all points of a curve coincide, if, and only if, the curve belongs to a linear complex.

\section{§5. Geometrical definition of the fundamental tetrahedron of reference.}

We have seen that there exists for every point of the curve $C_{y}$ a tetrahedron whose vertices $P_{y}, P_{z}, P_{\rho}, P_{\sigma}$ are determined by the choice of the independent variables $x$. In order that we may be able to obtain a clear insight into the geometry of the curve, it is necessary that we may be able to define this tetrahedron by purely geometrical considerations. As a consequence of our preceding results we are now able to do this.

We have already noticed that $P_{z}$ is a point on the tangent, and-by a properly chosen transformation $\xi=\xi(x)$ it may be transformed into any other point of the tangent. When the independent variable has been definitely chosen to be $x$, we obtain, therefore, a point $P_{z}$ on the tangent which is not, in general, distinguished by any geometrical property from any other point of the tangent. Its position may serve as a geometric image of the independent variable.

Consider the osculating conic

$$
x_{1}=0, \quad 40 x_{1} x_{3}-32 P_{2} x_{3}^{2}-15 x_{2}^{2}=0 .
$$

The polar of any point $\left(x_{1}^{\prime}, x_{2}^{\prime}, x_{3}^{\prime}, 0\right)$ of the osculating plane with respect to it, is the straight line

$$
x_{1}=0, \quad 20 x_{3}^{\prime} x_{1}-15 x_{2}^{\prime} x_{2}+\left(20 x_{1}^{\prime}-32 P_{2} x_{3}^{\prime}\right) x_{3}=0 .
$$


Therefore, the polar of $P_{z}$, whose coördinates are $(0,1,0,0)$, is the line $x_{2}=0, x_{4}=0$. In other words:

The line $P_{y} P_{p}$ is the polar of $P_{z}$ with respect to the osculating conic.

We shall speak of the curves $C_{z}, C_{\rho}, C_{\sigma}$ as the derivative curves of $C_{y}$ with respect to $x$, of the first, second and third kind respectively. The ruled surfaces which are obtained by joining the points of $C_{y}$ to the corresponding points of $C_{z}, C_{\rho}, C_{\sigma}$ shall be called derivative ruled surfaces of the first, second and third kind respectively. Then, the derivative ruled surface of the first kind is unique. It is simply the developable whose cuspidal edge is $C_{y}$. Let us consider the derivative ruled surface $S$ of the second kind generated by $P_{y} P_{\rho}$. The curve $C_{y}$ is, of course, an asymptotic curve upon it. This surface is characterized by the equations $(20)$, where $p_{1}$ has been assumed equal to zero.

According to the general theory of ruled surfaces, ${ }^{*}$ the asymptotic tangents to $S$ at the points $P_{y}$ and $P_{p}$ are obtained by joining these points to $2 z$ and

$$
2 \sigma-4 P_{2} z+2 P_{3} y
$$

respectively. Therefore the asymptotic tangent to $S$ at any point $\left(\alpha_{1}, 0, \alpha_{3}, 0\right)$ of $P_{y} P_{p}$ joins this point to

$$
2 P_{3} \alpha_{3} y+\left(2 \alpha_{1}-4 P_{2} \alpha_{3}\right) z+2 \alpha_{3} \sigma .
$$

Hence, the equation of the plane tangent to $S$ at $\left(\alpha_{1}, 0, \alpha_{3}, 0\right)$ is

$$
-\alpha_{3} x_{2}+\left(\alpha_{1}-2 P_{2} \alpha_{3}\right) x_{4}=0 \text {. }
$$

To the same point of $P_{y} P_{\rho}$ there corresponds a plane in the osculating linear complex. According to $(47 a)$ this is the plane

$$
\alpha_{3} x_{2}+\left(\alpha_{1}-2 P_{2} \alpha_{3}\right) x_{4}=0 \text {. }
$$

Therefore, if at any point of the generator of the derived ruled surface of the second kind we construct the tangent plane as well as the plane which corresponds to it in the osculating linear complex, these planes form an involution. The double planes of this involution are the osculating plane $\left(x_{4}=0\right)$, and a plane $\left(x_{2}=0\right)$ which contains $P_{\sigma}$, the point of the derivative curve of the third kind which corresponds to $P_{y}$.

The point which corresponds to this latter plane, is

$$
\beta=2 P_{2} y+\rho \text {. }
$$

According to $(47 a)$ we have further, corresponding to the point $P_{z}$ or $(0,1,0,0)$, the plane $x_{3}=0$, which also contains $P_{\sigma}$. The line $P_{y} P_{\sigma}$ is now completely determined, as follows :

* Wilczynski, Covariunts, etc. Transactions of the American Mathematical Society, vol. 3 (1902), p. 434. 
The generator of the derived ruled surface of the third kind is the intersection of the following two planes: 1st, the plane corresponding to $P_{z}$ in the osculating linear complex; $2 d$, that plane which is tangent to the derived ruled surface of the second kind at the same point which corresponds to it in the osculating linear complex.

It still remains to determine the position of $P_{\rho}$ and $P_{\sigma}$ on the lines $P_{y} P_{\rho}$ and $P_{y} P_{\sigma}$.

The osculating conic intersects $P_{y} P_{\rho}$ in $P_{y}$ and in $P_{a}$ where

$$
\alpha=4 P_{2} y+5 \rho \text {. }
$$

The cross-ratio of the four points $P_{y}, P_{\rho}, P_{a}, P_{\beta}$, is

$$
(\alpha, y, \beta, \rho)=\frac{5}{2} \text {. }
$$

If, upon the generator of the derived ruled surface of the second kind, there be marked its intersections with the osculating conic, and the point $P_{\beta}$ whose tangent plane coincides with the plane corresponding to it in the osculating linear complex, the point $\boldsymbol{P}_{\rho}$ is determined by the condition that the cross-ratio of these four points shall be equal to $\frac{5}{2}$.

If $P_{2}=0$ this definition of $P_{\rho}$ breaks down. In that case, however, $P_{a}$ and $P_{\beta}$ coincide with $P_{\rho}$. Therefore, if the derived ruled surface of the third kind is a developable, $P_{\rho}$ is that point on the generator of the derived surface of the second kind where this generator intersects the osculating conic the second time. At this point the plane, tangent to the ruled surface, and the plane, corresponding to it in the osculating linear complex, coincide.

If we use the notations of the theory of ruled surfaces,* we find from (20),

$$
\begin{gathered}
u_{12}=4, \quad u_{21}=8 P_{3}^{\prime}-4 P_{4}+8 P_{2}^{2}, \quad u_{11}-u_{22}=16 P_{2}, \\
\left(u_{11}-u_{22}\right)^{2}+4 u_{12} u_{21}=-64\left(P_{4}-2 P_{3}^{\prime}-6 P_{2}^{2}\right) .
\end{gathered}
$$

But $u_{11}-u_{22}=0$ is the condition that $C_{y}$ and $C_{\rho}$ shall be harmonically divided by the branches of the flecnode curves of the ruled surface, while $\left(u_{11}-u_{22}\right)^{2}+4 u_{12} u_{21}=0$ is the condition under which the two branches of the flecnode curve coincide. $\dagger$ Therefore, we obtain the following theorem.

If the derived ruled surface of the third kind is a developable, the intersections of the generator of the derived ruled surface of the second kind with the osculating conic give rise to two curves upon this surface harmonically conjugate with respect to the two branches of its flecnode curve.

If $\theta_{1}=0$, the second intersection of the generator of this surface with the osculating conic is a point of its flecnode curve. Moreover, the two branches of the flecnode curve must then coincide.

* Wilczynski, Covariants, etc., loc. cit., p. 445. Invariants, etc., Transactions of the American Mathematical Society, vol. 2 (1901), p. 6.

† Loc. cit., p. 444. 
It is to be noted that we have here a geometrical interpretation for the invariant equation $\theta_{4}=0$. We shall find two other, quite different interpretations for this condition later on. By the method of HALPHEN still another meaning can be attached to this equation, not however so purely geometrical.

We may, if we wish, make use of the torsal cubic in our further constructions. For, it is now defined entirely by geometrical considerations. If, in fact, we trace upon the developable, whose edge of regression is $C_{y}$ an arbitrary curve $C_{\bar{z}}$, we now know how the corresponding ruled surfaces of the second and third kind may be constructed. They depend upon an arbitrary function of $x$, as does the curve $C_{\bar{z}}$. Among the surfaces of the third kind there exists a single one-parameter family of developables. Upon that generator of each of these developables which passes through $P_{y}$ we mark the point where it intersects the cuspidal edge of the developable to which it belongs. The locus of these points is the torsal cubic.

We notice incidentally that the reduction of equation (1) to the LaguerreForsyth canonical form is equivalent to the determination of one of the developables of the third kind. Since this reduction is made by solving an equation of the RicCATi form we notice further the following theorem. The four curves on the developable of $C_{y}$ which correspond to any four of the developables of the third kind, intersect all of the tangents of $C_{y}$ in point-rows of the same cross-ratio.

Let us consider the developable surface of the torsal cubic, which is given by equations (44). We are going to find its intersection with the plane $P_{y} P_{z} P_{\sigma}$, or $x_{3}=0$. The intersection of the plane $u_{1}, \ldots, u_{4}$, which osculates the cubic at the point whose parameter is $\eta$, with the plane $x_{3}=0$ is the line

$$
-180 x_{1}+270 \eta x_{2}+\left(540 \theta_{3}+54 P_{2}^{\prime}-324 P_{2} \eta+135 \eta^{3}\right) x_{4}=0
$$

of this plane. As $\eta$ changes this line envelops a curve, the required intersection. Its equation will be found by eliminating $\eta$ between the above equation and this other one

$$
270 x_{2}+\left(-324 P_{2}+405 \eta^{2}\right) x_{4}=0
$$

obtained from it by differentiation with respect to $\eta$. This elimination may be easily performed. The result is

$$
F=8\left(5 x_{2}-6 x_{4}\right)\left(5 x_{1}-6 x_{4}\right)^{2}+15 x_{4}\left\{10 x_{1}-\left(30 \theta_{3}+3 P_{2}^{\prime}\right) x_{4}\right\}^{2}=0 .
$$

This plane cubic together with the tangent $P_{y} P_{z}$ gives the complete intersection of the plane $P_{y} P_{z} P_{\sigma}$ with the developable of the torsal cubic. It has a cusp at $P_{z}$, and the equation of its cusp tangent is

$$
5 x_{1}-6 x_{4}=0
$$


as one may find by the general theory of plane curves. It intersects $P_{y} P_{\sigma}$ in the point

$$
\kappa=24 y+20 \sigma .
$$

The tangent to the plane cubic at $P_{y}$ is

$$
10 x_{2}+3 x_{4}=0 \text {. }
$$

It intersects the cubic again in the point

$$
\left(12+30 \theta_{3}+3 P_{2}^{\prime}\right) y-6 z+20 \sigma .
$$

If this point be joined to $P_{z}$ by a straight line, the latter will intersect $P_{y} P_{\sigma}$ in the point

$$
\lambda=\left(12+30 \theta_{3}+3 P_{2}^{\prime}\right) y+20 \sigma .
$$

The plane $P_{y} P_{z} P_{\sigma}$ is tangent to the torsal cubic. It intersects it once more in the point corresponding to $\eta=0$, viz., $\left(60 \theta_{3}+6 P_{2}^{\prime}\right) y+24 P_{2} z+20 \sigma$. A line joining this point to $P_{z}$ intersects $P_{y} P_{\sigma}$ in

$$
\mu=\left(60 \theta_{3}+6 P_{2}^{\prime}\right) y+20 \sigma .
$$

Consider the four points $P_{\kappa}, P_{\lambda}, P_{\mu}$ and $P_{y}$. We have

$$
\lambda=\frac{\mu+\kappa}{2}, \quad\left(30 \theta_{3}+3 P_{2}^{\prime}-12\right) y=\frac{\mu-\kappa}{2} ;
$$

so that $P_{\lambda}$ is the harmonic conjugate of $P_{y}$ with respect to $P_{\kappa}$ and $P_{\mu}$.

The osculating cubic differs from the torsal cubic only in having $2\left(P_{3}-P_{2}^{\prime}\right)$ in place of $10 \theta_{3}+P_{2}^{\prime}$. Consequently the plane cubic in which its developable intersects the plane $P_{y} P_{z} P_{\sigma}$ is

$$
\bar{F}=8\left(5 x_{2}-6 x_{4}\right)\left(5 x_{1}-6 x_{4}\right)^{2}+15 x_{4}\left\{10 x_{1}-6\left(P_{3}-P_{2}^{\prime}\right) x_{4}\right\}^{2}=0
$$

If we denote by $P_{\bar{\kappa}}, P_{\bar{\lambda}}, P_{\bar{\mu}}$ the points constructed with respect to this curve in the same way as $P_{\kappa}$, and $P_{\lambda}$ and $P_{\mu}$ were with respect to $F=0$, we find

$$
\bar{\kappa}=\kappa, \bar{\lambda}=\left(12+6 P_{3}-6 P_{2}^{\prime}\right) y+20 \sigma, \bar{\mu}=\left(12 P_{3}-12 P_{2}^{\prime}\right) y+20 \sigma
$$

the cusp and its tangent being common to the two curves, as well as the tangent at $P_{y}$. I refrain from formulating explicitly the various theorems which may be obtained from these equations.

In order to obtain a simple construction for $P_{\sigma}$, we shall consider finally the developable generated by the motion. of the plane $P_{y} P_{\rho} P_{\sigma}$. The equation of this plane is $x_{2}=0$. As $x$ changes into $x+\delta x, y, z, \rho$ change into $y+y^{\prime} \delta x$, $\rho+\rho^{\prime} \delta x, \sigma+\sigma^{\prime} \delta x$ respectively, where $y^{\prime}, \rho^{\prime}, \sigma^{\prime}$ are given by equations (5). The equation of the plane of these points, referred to the tetrahedron $P_{y}, P_{z}, P_{\rho}, P_{\sigma}$, is 


$\left|\begin{array}{cccc}x_{1} & x_{2} & x_{3} & x_{4} \\ 1-p_{1} \delta x & \delta x & 0 & 0 \\ -\left(P_{3}-P_{2}^{\prime}\right) \delta x & -2 P_{2} \delta x & 1-p_{1} \delta x & \delta x \\ -\left(P_{4}-P_{3}^{\prime}\right) \delta x & -3\left(P_{3}-P_{2}^{\prime}\right) \delta x & -3 P_{2} \delta x & 1-p_{1} \delta x\end{array}\right|=0$,

which becomes, when developed,

$$
x_{1} \delta x-x_{2}\left(1-3 p_{1} \delta x\right)-2 x_{3} P_{2} \delta x-3 x_{4}\left(P_{3}-P_{2}^{\prime}\right) \delta x=0 .
$$

Therefore the equations of the generator of the developable, generated by the motion of the plane $P_{y} P_{\rho} P_{\sigma}$, are

$$
x_{2}=0, \quad x_{1}-2 P_{2} x_{3}-3\left(P_{3}-P_{2}^{\prime}\right) x_{4}=0 .
$$

It intersects the generator $P_{y} P_{p}\left(x_{2}=x_{4}=0\right)$ of the derived ruled surface of the second kind in that point

$$
\beta=2 P_{2} y+\rho
$$

whose tangent plane coincides with the plane corresponding to it in the osculating linear complex. Its intersection with $P_{y} P_{\sigma}$, the generator of the derived ruled surface of the third kind, is

$$
\gamma=3\left(P_{3}-P_{2}^{\prime}\right) y+\sigma .
$$

The generator of the developable joins $P_{\beta}$ to $P_{\gamma}$. We wish to determine its edge of regression. If

$$
\delta=l \beta+m \gamma
$$

is the point where $P_{\beta} P_{\gamma}$ meets the edge of regression, we must have

$$
\delta^{\prime}=r \beta+s \gamma, \quad \text { or } \quad l \beta^{\prime}+m \gamma^{\prime}=\bar{r} \beta+\bar{s} \gamma .
$$

We proceed to determine the ratio of $l$ to $m$. We find

$$
\begin{aligned}
& \beta^{\prime}=\left(3 P_{2}^{\prime}-2 p_{1} P_{2}-P_{3}\right) y-p_{1} \rho+\sigma, \\
& \gamma^{\prime}=-\left[P_{4}-\dot{4} P_{3}^{\prime}+3 P_{2}^{\prime \prime}+3 p_{1}\left(P_{3}-P_{2}^{\prime}\right)\right] y-3 P_{2} \rho-p_{1} \sigma .
\end{aligned}
$$

We may eliminate $\rho$ and $\sigma$ by (52) and (61). This gives

$$
\begin{aligned}
& \beta^{\prime}=-4 \theta_{3} y-p_{1} \beta+\gamma, \\
& \gamma^{\prime}=-\left(P_{4}-4 P_{3}^{\prime}+3 P_{2}^{\prime \prime}-6 P_{2}^{2}\right) y-3 P_{2} \beta-p_{1} \gamma .
\end{aligned}
$$

We may therefore put

so that

$$
l=P_{4}-4 P_{3}^{\prime}+3 P_{2}^{\prime \prime}-6 P_{2}^{2}, \quad m=-4 \theta_{3} ;
$$

$$
\delta=\left(P_{4}-4 P_{3}^{\prime}+3 P_{2}^{\prime \prime}-6 P_{2}^{2}\right) \beta-4 \theta_{3} \gamma
$$

gives the edge of regression. This gives the following theorem: 
The developable, generated by the plane of the generators of the derived ruled surfaces of the second and third kind, has its edge of regression upon the derived ruled surface of the second kind, if, and only if, the curve $C_{y}$ belongs to a linear complex.

We may write in place of $(51)$

We have from (59)

$$
\gamma=60\left(P_{3}-P_{2}^{\prime}\right) y+20 \sigma .
$$

$$
\bar{\mu}=12\left(P_{3}-P_{2}^{\prime}\right) y+20 \sigma \text {. }
$$

Therefore, the cross-ratio of the four points $P_{\gamma}, P_{y}, P_{\bar{\mu}}$ and $P_{\sigma}$ is

$$
(\gamma, y, \bar{\mu}, \sigma)=5 \text {. }
$$

We have found finally a geometrical definition for $P_{\sigma}$, which we may recapitulate as follows. The plane of the tangent and the generator of the third derived ruled surface intersects the osculating cubic in $P_{y}$ counted twice and one other point. If the latter point be joined to $P_{z}$ by a straight line we obtain a certain point $P_{\bar{\mu}}$ as the intersection of this line with $P_{y} P_{\sigma} . \quad$ The generator of the developable, generated by the plane of the generators of the derived ruled surfaces of the second and third kind, intersects $P_{y} P_{\sigma}$ in another point $P_{\gamma} \cdot P_{\sigma}$ may now be found as that point of $P_{y} P_{\sigma}$ which makes the cross-ratio

$$
\left(P_{\gamma}, P_{y}, P_{\bar{\mu}}, P_{\sigma}\right)=5 \text {. }
$$

We have shown how to construct the fundamental tetrahedron when $P_{z}$ is given. If $P_{\rho}$ is given, $P_{z}$ can be found at once as the pole of $P_{y} P_{\rho}$ with respect to the osculating conic. If $P_{\sigma}$ is given we may find first its polar plane with respect to the osculating linear complex which is

$$
-x_{1}+2 P_{2} x_{3}=0
$$

and therefore passes through $P_{z}$, but not through $P_{y} . P_{z}$ can therefore be found at once at the intersection of this plane with the tangent to $C_{y}$ at $P_{y}$.

We see therefore, that any one of the three points $P_{z}, P_{\rho}, P_{\sigma}$ determines uniquely the others.

$\S 6$. Some further properties of the derived ruled surfaces of the second and third kind.

Let us suppose $p_{1}=P_{2}=0$, so that the derived ruled surface of the third kind is a developable, and let us consider the derived ruled surface of the second kind which corresponds to it. We proceed to deduce the equation of its osculating linear complex.

Let $Y$ and $R$ denote the developments of $y$ and $\rho$ in the vicinity of the ordi- 
nary point $x=a$, and replace again $x-a$ by $x$ in the developments. Then we shall find

where

$$
Y=y_{1} y+y_{2} z+y_{3} \rho+y_{4} \sigma, \quad R=\rho_{1} y+\rho_{2} z+\rho_{3} \rho+\rho_{4} \sigma,
$$

$$
\begin{aligned}
& \rho_{1}=-P_{3} x-\frac{1}{2} P_{4} x^{2}-\frac{1}{6} P_{4}^{\prime} x^{3}+\frac{1}{24}\left(4 P_{3}^{2}-P_{4}^{\prime \prime}\right) x^{4}+\cdots, \\
& \rho_{2}=-2 P_{3} x^{2}-\frac{1}{6}\left(4 P_{3}^{\prime}+P_{4}\right) x^{3}-\frac{1}{12}\left(2 P_{3}^{\prime \prime}+P_{4}^{\prime}\right) x^{4}+\cdots, \\
& \rho_{3}=1-\frac{2}{3} P_{3} x^{3}-\frac{1}{2} 4\left(8 P_{3}^{\prime}+P_{4}\right) x^{4}+\cdots, \\
& \rho_{4}=x-\frac{1}{6} P_{3} x^{4}+\cdots,
\end{aligned}
$$

while $y_{1}, \cdots, y_{4}$ have been computed before. Denote by

$$
\omega_{i k}=y_{i} \rho_{k}-y_{k} \rho_{i}
$$

the Plückerian coördinates of the line joining the two points. We find

Let

$$
\begin{aligned}
& \omega_{12}=-P_{3} x^{2}-\frac{1}{3}\left(2 P_{3}^{\prime}-P_{4}\right) x^{3}-\frac{1}{12}\left(2 P_{3}^{\prime \prime}-P_{4}^{\prime}\right) x^{4}+\cdots, \\
& \omega_{13}=1-\frac{1}{3} P_{3} x^{3}-\frac{1}{6}\left(2 P_{3}^{\prime}-P_{4}\right) x^{4}+\cdots, \\
& \omega_{14}=x-\frac{1}{6} P_{3} x^{4}+\cdots, \\
& \omega_{23}=x+\frac{1}{6} P_{3} x^{4}+\cdots, \\
& \omega_{24}=x^{2}+\cdots, \quad \omega_{34}=\frac{1}{3} x^{3}+\cdots
\end{aligned}
$$

$$
a \omega_{12}+b \omega_{12}+c \omega_{14}+d \omega_{23}+e \omega_{24}+f \omega_{34}=0
$$

be the equation of the osculating linear complex of the surface in question. Then, the coefficients of all powers of $x$ up to and including $x^{4}$ must be zero, if we substitute the above developments of $\omega_{i k}$ into the left member. This gives us the following equations :

$$
\begin{array}{r}
b=0, \quad c+d=0, \quad-P_{3} a+e=0, \\
-\frac{1}{3}\left(2 P_{3}^{\prime}-P_{4}\right) a-\frac{1}{3} P_{3} b+\frac{1}{3} f=0, \\
-\frac{1}{12}\left(2 P_{3}^{\prime \prime}-P_{4}^{\prime}\right) a-\frac{1}{6}\left(2 P_{3}^{\prime}-P_{4}\right) b-\frac{1}{6} P_{3} c+\frac{1}{6} P_{3} d=0,
\end{array}
$$

whence the ratios of the coefficients may be easily deduced.

We find thus the equation of the linear complex osculating the derived ruled surface of the second kind which corresponds to a developable of the third kind; it is

$(64)-4 P_{3} \omega_{12}-\left(P_{4}^{\prime}-2 P_{3}^{\prime \prime}\right)\left(\omega_{14}-\omega_{23}\right)-4 P_{3}^{2} \omega_{24}+4\left(P_{4}-2 P_{3}^{\prime}\right) P_{3} \omega_{34}=0$.

It coincides with the osculating linear complex of $C_{y}$ if and only if $P_{3}=0$, i. e., if $C_{y}$ belongs to a linear complex. This result is also obvious for geometrical reasons. 
The coördinates $v_{k}$ of the plane, which corresponds to a point $x_{1}, x_{2}, x_{3}, x_{4}$ in the linear complex (64), are given by

$$
\begin{aligned}
& \omega v_{1}=4 P_{3} x_{2}+\left(P_{4}^{\prime}-2 P_{3}^{\prime \prime}\right) x_{4}, \\
& \omega v_{2}=-4 P_{3} x_{1}-\left(P_{4}^{\prime}-2 P_{3}^{\prime \prime}\right) x_{3}+4 P_{3}^{2} x_{4}, \\
& \omega v_{3}=\left(P_{4}^{\prime}-2 P_{3}^{\prime \prime}\right) x_{2}-4 P_{3}\left(P_{4}-2 P_{3}^{\prime}\right) x_{4}, \\
& \omega v_{4}=-\left(P_{4}^{\prime}-2 P_{3}^{\prime \prime}\right) x_{1}-4 P_{3}^{2} x_{2}+4 P_{3}\left(P_{4}-2 P_{3}^{\prime}\right) x_{3},
\end{aligned}
$$

where $\omega$ is a proportionality factor.

Let us consider at the same time the osculating linear complex of $C_{y}$. The lines common to the two complexes form a congruence whose directrices we propose to find. This we can do quite easily by writing down the equations which express that, for a point on one of the directrices, the two planes corresponding to it in the two complexes must coincide. The right members of (65) must, for such a point be equal to

$$
-\omega x_{1}, \quad+\omega x_{3}, \quad-\omega x_{2}, \quad+\omega x_{1}
$$

respectively, where $\omega$ is a proportionality factor.

The four equations obtained in this way can be satisfied only if their determinant vanishes, which gives

$$
2 P_{3}^{\prime \prime}-P_{4}^{\prime}-\omega= \pm 4 P_{3} v^{\prime} \overline{2} \bar{P}_{3}^{\prime}-P_{4},
$$

whence the following equations for the two directrices

$$
\begin{array}{r} 
\pm \sqrt{2 P_{3}^{\prime}-P_{4}} x_{1}-P_{3} x_{2}-\left(2 P_{3}^{\prime}-P_{4}\right) x_{3}=0, \\
-x_{2} \pm \sqrt{2} P_{3}^{\prime}-P_{4} x_{4}=0, \\
-x_{1} \pm \sqrt{2 P_{3}^{\prime}-P_{4}} x_{3}+P_{3} x_{4}=0,
\end{array}
$$

of which three equations only two are independent, and where we have assumed $P_{3} \neq 0$. In fact, if $P_{3}$ were zero the two complexes would coincide and the congruence would be indeterminate.

Since we have assumed $P_{2}=0$, the quantity under the square root is $-\theta_{4}$. We find a second interpretation for the condition $\theta_{4}=0$. If $\theta_{4}=0$, the congruence has coincident directrices. We may combine this with our former result to the following theorem.

Choose as derived ruled surface of the third kind one of the developables of the single family which exists. Consider the osculating linear complex of the corresponding ruled surface $S$ of the second kind. Let the directrices of the congruence, which this complex has in common with the osculating linear complex of the fundamental curve $C_{y}$, coincide. Then the two branches of the 
flecnode curve of $S$ coincide and the generator of $S$ which passes through $P_{y}$ will intersect the osculating conic of $C_{y}$ in $P_{y}$, and a second point whose locus is the flecnode curve of the surface $S$.

I refrain from formulating the converse. The above conditions are fulfilled if and only if $\theta_{4}=0$.

Let us.consider one of the directrices (67), for example that one which corresponds to the plius sign of the square root. Then we see that

$$
\begin{aligned}
& \alpha=P_{3} \sqrt{2 P_{3}^{\prime}-P_{4}} y+P_{3} \rho, \\
& \beta=\sqrt{2 P_{3}^{\prime}-P_{4}}\left(P_{3} y+\sqrt{2 P_{3}^{\prime}-P_{4}} z+\sigma\right)
\end{aligned}
$$

are two points on the directrix. We have multiplied each expression by a factor so as to have $\alpha$ and $\beta$ of the same weight. If now we change the independent variable, but in such a way as not to disturb the condition $P_{2}=0$, we shall get in (64) a single infinity of complexes, and in (67) two families of lines, the directrices of the single infinity of congruences which thus result. We are going to study to some extent the two ruled surfaces thus generated.

Put

$$
k=\sqrt{2 P_{3}^{\prime \prime}-P_{4}} .
$$

Making the transformations which preserve $P_{2}=0$, for which we must have

$$
\mu=\eta^{\prime}-\frac{1}{2} \eta^{2}=0,
$$

we find that $\alpha$ and $\beta$ are transformed into $\bar{\alpha}$ and $\bar{\beta}$, where

$$
\begin{aligned}
& \left(\xi^{\prime}\right)^{5} \bar{\alpha}=\alpha+2 \eta P_{3} z+\frac{3}{2} \eta^{2} P_{3} y, \\
& \left(\xi^{\prime}\right)^{5} \bar{\beta}=\beta+\frac{3}{2} \frac{k}{P_{3}} \eta \alpha+k\left(\frac{3}{2} \eta^{2} z+\frac{3}{4} \eta^{3} y\right) .
\end{aligned}
$$

The point $m \bar{\alpha}+n \bar{\beta}$ will be an-arbitrary point on the line joining $P_{\bar{\alpha}} P_{\bar{\beta}}$. We find therefore, the equations of our surface referred to two parameters $\eta$ and $m / n$,

$$
\begin{aligned}
& x_{1}=\frac{3}{2} m P_{3} \eta^{2}+(m+n) P_{3} k+n\left(\frac{3}{2} \eta k^{2}+\frac{3}{4} \eta^{3} k\right), \\
& x_{2}=2 m \eta P_{3}+n\left(k^{2}+\frac{3}{2} \eta^{2} k\right), \\
& x_{3}=m P_{3}+\frac{8}{2} k n \eta, \\
& x_{4}=n k .
\end{aligned}
$$

The nature of this surface may be easily determined. We have, returning to the two curves $C_{a}$ and $C_{\beta}$ upon it, for $C_{a}$

$$
\alpha_{1}=k+\frac{3}{2} \eta^{2}, \quad \alpha_{2}=2 \eta, \quad \alpha_{3}=1, \quad \alpha_{4}=0,
$$

if we put $n=0, m=1 / P_{3}$ in (70). If we put $m=0, n=1 / k$ we find for $C_{\beta}$

$$
\beta_{1}=P_{3}+\frac{8}{2} k \eta+\frac{3}{4} \eta^{3}, \quad \beta_{2}=k+\frac{8}{2} \eta^{2}, \quad \beta_{3}=\frac{8}{2} \eta, \quad \beta_{4}=1 .
$$


But $\left(\alpha_{k}, \beta_{k}\right)$ are simultaneous solutions of the equation

$$
\frac{d \beta}{d \eta}={ }_{2}^{3} \alpha,
$$

which proves that the ruled surface which we are considering is a developable whose edge of regression is the twisted cubic $C_{\beta}$.

The curve $C_{a}$ is a conic, the intersection of the developable of the cubic with the osculating plane of $C_{y}$. Its equations are

$$
x_{4}=0, \quad-3 x_{2}^{2}+8 x_{1} x_{3}-8 k x_{3}^{2}=0 .
$$

We notice that for $\theta_{4}=0$ it coincides with the osculating conic, a further interpretation of this condition. In general, the two conics have a contact of third order at $\boldsymbol{P}_{y}$.

If, in these equations, we change $k$ into $-k$ we obtain the developable, cubic and conic associated with the second directrix of our congruence. A considerable number of other configurations are suggested by the combinations of these various curves and surfaces. I will refrain, however, from any further study in this direction.

The curve $C_{y}$ is an asymptotic curve upon every derived ruled surface of the second kind. Moreover, the most general derived ruled surface of the second kind depends upon one arbitrary function, as does also the most general ruled surface containing $C_{y}$ as an asymptotic curve. It is easy to see that the derived ruled surface of the second kind may be made to coincide with any ruled surface upon which $C_{y}$ is an asymptotic curve if the independent variable be properly chosen.

Upon the derived ruled surface of the third kind, $C_{y}$ can never be an asymptotic curve. It may, however, be one branch of the flecnode curve. In fact, if we form the quantities $u_{i k}$ of the theory of ruled surfaces for system (27), we find

$$
u_{12}=-\frac{P_{2}^{\prime}}{P_{2}^{2}}
$$

But $u_{12}=0$ is the condition that $C_{y}$ may be a branch of the flecnode curve on the surface generated by $P_{y} P_{\sigma}$. Suppose that the variable has been so chosen as to make $P_{2}^{\prime}=0$. The most general transformation which is possible, leaving this relation invariant, must, according to (11) satisfy the condition

or

$$
-2 \eta P_{2}-\frac{5}{6} \mu^{\prime}+\frac{5}{3} \eta \mu=0,
$$

a differential equation of the second order for $\eta$. Moreover two different solutions of this equation always give rise to two.distinct ruled surfaces. For, let 
$\eta_{1}$ and $\eta_{2}$ be two such solutions, and let $\sigma_{1}, \sigma_{2}$ be the corresponding values of $\sigma$. Then, according to (9),

$$
\sigma_{\kappa}=\frac{1}{\left(\xi_{\kappa}^{\prime}\right)^{3}}\left[\sigma+{ }_{2}^{3} \eta_{\kappa} \rho+\left(\mu_{\kappa}+{ }_{2}^{3} \eta_{\kappa}^{2}\right) z+\frac{1}{4}\left(\mu_{\kappa}^{\prime}+4 \eta_{\kappa} \mu_{\kappa}+3 \eta_{\kappa}^{3}\right) y\right] \quad(\kappa=1,2),
$$

But if the same ruled surface corresponds to $\eta_{1}$ and $\eta_{2}$, the three points $y, \sigma_{1}$ and $\sigma_{2}$ must be collinear. We must therefore be able to reduce

$$
\left(\xi_{1}^{\prime}\right)^{3} \sigma_{1}-\left(\xi_{2}^{\prime}\right)^{3} \sigma_{2}=\frac{3}{2}\left(\eta_{1}-\eta_{2}\right) \rho+\cdots
$$

to a multiple of $y$. But this is clearly only possible if $\eta_{2}=\eta_{1}$.

We see, therefore, that there are $\infty^{2}$ derived ruled surfuces of the third kind upon which $C_{y}$ is one branch of the flecnode curve.

If $P_{2}^{\prime}$ is not zero, our problem leads to the differential equation

$$
\frac{d}{d x}\left(\eta^{\prime}-\frac{1}{2} \eta^{2}\right)=\frac{6}{5} P_{2}^{\prime}-\frac{12}{5} P_{2} \eta+2 \eta\left(\eta^{\prime}-\frac{1}{2} \eta^{2}\right),
$$

which is of the second order and third degree.

I have shown in a former paper that the most general ruled surface which has $C_{y}$ as one branch of its flecnode curve contains an arbitrary function in its general expression.* I have also shown that together with any such surface, its flecnode surface and each member of a single infinity of surfaces determined by these two, also contains $C_{y}$ as one branch of its flecnode curve. One might imagine that there could be based upon these theorems a transformation theory of equation (72). This is not the case however. For, if one of the surfaces containing $C_{y}$ as a branch of its flecnode curve is a derived ruled surface of the third kind, its flesnode surface is not, nor is any member of the family of ruled surfaces just mentioned.

Corresponding to the $\infty^{2}$ solutions of (72) or of

$$
\mu^{\prime}=\frac{6}{5} P_{2}^{\prime}-\frac{1}{6} \frac{2}{2} P_{2} \eta+2 \eta \mu,
$$

we find $\infty^{2}$ positions for $P_{\bar{\sigma}}$, viz.:

$$
4\left(\xi^{\prime}\right)^{3} \bar{\sigma}=\left(\frac{6}{6} P_{2}^{\prime}-\frac{12}{5} P_{2} \eta+6 \eta \mu+3 \eta^{3}\right) y+\left(4 \mu+6 \eta^{2}\right) z+6 \eta \rho+4 \sigma .
$$

The locus of these points is a cubic surface

$$
27 P_{2}^{\prime} x_{4}^{3}-36 P_{2} x_{3} x_{4}^{2}+90 x_{2} x_{3} x_{4}-90 x_{1} x_{4}^{2}-40 x_{3}^{3}=0,
$$

which contains $P_{y} P_{z}$, the tangent of $C_{y}$ as a double line. It is therefore a ruled surface. It is in fact a CAYLEY's cubic scroll.

If one derived surface of the third kind is known upon which $C_{y}$ is a branch

* General theory of curves on ruled surfaces, Transactions of the American Mathematical Society, vol. 6 (1905), p. 78. 
of the flecnode curve, two others may be found by merely solving a quadratic equation.

In fact, suppose that a solution $\eta$ of (72) be known. We may make a transformation of the independent variables, $\xi=\xi(x)$ such that

$$
\begin{aligned}
& \xi^{\prime \prime} \\
& \xi^{\prime}
\end{aligned}=\eta \text {. }
$$

In the resulting equation $\overline{P_{2}^{\prime}}=0$. If we again denote the independent variable by $x,(72)$ becomes

$$
\eta^{\prime \prime}-\eta \eta^{\prime}=-\frac{12}{5} P_{2} \eta+2 \eta\left(\eta^{\prime}-\frac{1}{2} \eta^{2}\right),
$$

where $P_{2}$ is a constant, since $P_{2}^{\prime}=0$. But we may satisfy this equation by putting $\eta=$ const., which gives the equation

whence

$$
\eta^{3}+\frac{12}{5} P_{2} \eta=0
$$

$$
\eta=0, \quad \pm \sqrt{-\frac{12}{3}-P_{2}} \text {. }
$$

The root $\eta=0$ gives the original solution. The other two are new.

\$7. The principal tangent plane of two space curves. The covariants. Transition to Halphen's investigations.

HALPHEN has introduced a very important notion, which we shall now proceed to explain.

Let there be given two space curves having at a point $P$ a contact of the $n$th order. If these curves be projected from any center $Q$ upon a plane, the projections will also have, in general, a contact of the $n$th order at the point corresponding to $P$. HALPHEN shows that there exists a plane passing through the common tangent of the two curves such that if the center of projection be taken anywhere within it, the contact of the projections will be of an order higher than $n$. This plane he calls the principal tangent plane of the two curves. *

We shall follow HALPHEN in determining the principal tangent plane at $P_{y}$ of the curve $C_{y}$ and its osculating cubic. This will lead us to an especially simple form for the development of the equations of the curve, which is also due to HALPHEN and on the basis of which he draws his further conclusions. It will also enable us to substitute for our system of covariants $C_{2}, C_{3}, C_{4}$ another system whose geometrical significance will be apparent, and in terms of which $C_{2}, C_{3}, C_{4}$ may be expressed.

* Halphen, Sur les invariants différentiels des courbes gauches, Journal de l'Ecole Polytechnique, vol. 47 (1880), p. 25. 
Assuming $P_{2}=0$, the equations of the osculating cubic, referred to the tetrahedron $P_{y} P_{*} P_{p} P_{\sigma}$, are

Let us put

$$
3 x_{2} x_{4}-2 x_{3}^{2}=0, \quad 2 x_{3}\left(5 x_{1}-3 P_{3} x_{4}\right)-5 x_{2}^{2}=0 .
$$

$$
\bar{x}_{1}=x_{2}, \quad \bar{x}_{2}=\frac{2}{5} x_{3}, \quad \bar{x}_{3}=\frac{6}{25} x_{4}, \quad \bar{x}_{4}=5 x_{1}-3 P_{3} x_{4},
$$

and

$$
x=\frac{\bar{x}_{1}}{\bar{x}_{4}}, \quad y=\frac{\bar{x}_{2}}{\bar{x}_{4}}, \quad z=\frac{\bar{x}_{3}}{\bar{x}_{4}},
$$

Then the equations of the cubic reduce to

$$
y=x^{2}, \quad z=x^{3} .
$$

The relation of the new tetrahedron of reference to the cubic is quite simple. The plane $\bar{x}_{3}=0$ is the osculating plane at $P ; \bar{x}_{2}=0$ is some other plane through the tangent; this plane intersects the cubic in another point $Q$; the plane tangent to the cubic at $Q$ and passing through $P$ is $\bar{x}_{1}=0$; the osculating plane at $Q$ is $\bar{x}_{4}=0$.

Since $\bar{x}_{2}=0$ may be chosen in an infinity of ways we see that the reduction of the equations of a space cubic to the form (76) may be accomplished in an infinity of ways.

For the curve $C_{y}$ we have

$$
\begin{aligned}
& y_{1}=1-\frac{P_{3}}{3 !} x^{3}-\frac{P_{4}}{4 !} x^{4}-\frac{P_{4}^{\prime}}{5 !} x^{5}-\frac{P_{4}^{\prime \prime}-4 P_{3}^{2}}{6 !} x^{6} \\
& -\frac{P_{4}^{(3)}-5 P_{3} P_{4}-12 P_{3} P_{3}^{\prime}}{7 !} x^{7}+\cdots, \\
& y_{2}=x-\frac{P_{3}}{3 !} x^{4}-\frac{P_{4}+4 P_{3}^{\prime}}{5 !} x^{5}-\frac{2 P_{4}^{\prime}+4 P_{3}^{\prime \prime}}{6 !} x^{6} \\
& +\frac{16 P_{3}^{2}-3 P_{4}^{\prime \prime}-4 P_{3}^{(3)}}{7 !} x^{7}+\cdots, \\
& y_{3}=\frac{1}{2} x^{2}-\frac{4 P_{3}}{5 !} x^{5}-\frac{1}{6 !}\left(P_{4}+8 P_{3}^{\prime}\right) x^{6}-\frac{1}{7 !}\left(3 P_{4}^{\prime}+12 P_{3}^{\prime \prime}\right) x^{7}+\cdots, \\
& y_{4}=\frac{1}{6} x^{3}-\frac{4}{6 !} P_{3} x^{6}-\frac{1}{7 !}\left(P_{4}+12 P_{3}^{\prime}\right) x^{7} \cdots
\end{aligned}
$$

If we refer it to the same tetrahedron to which we have just referred the osculating cubic, and if we put

$$
\xi=\frac{\bar{y}_{1}}{\bar{y}_{4}}, \quad \eta=\frac{\bar{y}_{2}}{\bar{y}_{4}}, \quad \zeta=\frac{\bar{y}_{3}}{\bar{y}_{4}},
$$


we shall find

$$
\begin{aligned}
& \eta=\xi^{2}+\frac{125}{36}\left(8 P_{3}^{\prime}-5 P_{4}\right) \xi^{6}+{ }_{7.36}^{625}\left(8 P_{3}^{\prime \prime}-5 P_{4}^{\prime}\right) \xi^{7}+\cdots \\
& \zeta=\xi^{3}-\frac{25}{3} P_{3} \xi^{6}+\frac{125}{7.12}\left(36 P_{3}^{\prime}-25 P_{4}\right) \xi^{7}+\cdots
\end{aligned}
$$

If we put

$$
\xi=\epsilon X, \quad \eta=\epsilon^{2} Y, \quad \zeta=\epsilon^{3} Z, \quad \epsilon^{3}=-\frac{3}{25 P_{3}},
$$

assuming, therefore, that $P_{3}$ is not zero, this becomes

where

$$
\begin{aligned}
& Y=X^{2}+\lambda_{6} X^{6}+\lambda_{7} X^{7}+\cdots, \\
& Z=X^{3}+\mu_{6} X^{6}+\mu_{7} X^{7}+\cdots,
\end{aligned}
$$

$$
\begin{array}{ll}
\lambda_{6}=\frac{125}{36}\left(8 P_{3}^{\prime}-5 P_{4}\right) \epsilon^{4}, & \lambda_{7}=\frac{625}{7.36}\left(8 P_{3}^{\prime \prime}-5 P_{4}^{\prime}\right) \epsilon^{5}, \\
\mu_{6}=1, & \mu_{7}=\frac{125}{7.12}\left(36 P_{3}^{\prime}-25 P_{4}\right) \epsilon^{4} .
\end{array}
$$

But we can make a further change of coördinates without disturbing the form (80) of the development. For the plane $\bar{x}_{2}=0$ may be taken to be any plane through the tangent. It may be taken in such a way as to make $\lambda_{6}$ vanish, as we shall presently see. The plane thus obtained will obviously be the principal tangent plane of the curve $C_{y}$ and its osculating cubic.

Instead of working out the transformation geometrically we shall put with HALPHEN

where

$$
\bar{X}=\frac{\xi_{1}}{\omega_{1}}, \quad \bar{Y}=\frac{\eta_{1}}{\omega_{1}}, \quad \bar{Z}=\frac{Z_{1}}{\omega_{1}},
$$

$$
\begin{aligned}
& \omega_{1}=1+3 p X+3 p^{2} Y+p^{3} Z, \quad \eta_{1}=Y+p Z, \\
& \xi_{1}=X+2 p Y+p^{2} Z,
\end{aligned}
$$

the quantity $p$ being arbitrary. We shall find with him

$$
\begin{aligned}
& \bar{Y}=\bar{X}^{2}+\Lambda_{6} \bar{X}^{6}+\Lambda_{7} \bar{X}^{7}+\cdots, \\
& \bar{Z}=\bar{X}^{3}+M_{6} \bar{X}^{6}+M_{7} \bar{X}^{7}+\cdots,
\end{aligned}
$$

as the result of applying this transformation to any system of equations of the form (80). The coefficients of (83) are

For

$$
\begin{array}{ll}
M_{6}=\mu_{6}, & M_{7}=\mu_{7}+3 p \mu_{6}, \\
\Lambda_{6}=\lambda_{6}+p \mu_{6}, & \Lambda_{7}=\lambda_{7}+p \mu_{7}+p\left(p \mu_{7}-\lambda_{6}\right) . \\
& p=-\frac{\lambda_{6}}{\mu_{6}}
\end{array}
$$


this reduces to the canonical form required, in which we may also assume $\mu_{6}=1$ if the curve does not belong to a linear complex.

If we combine the various transformations which we have made successively we get the following result: If we introduce non-homogeneous coördinates by putting

$$
X=\frac{\bar{y}_{1}}{\bar{y}_{4}} \cdot \quad Y=\frac{\bar{y}_{2}}{\bar{y}_{4}}, \quad Z=\frac{\bar{y}_{3}}{\bar{y}_{4}},
$$

where

$$
\begin{aligned}
& \bar{y}_{1}=25 \epsilon^{2} y_{2}+20 p \epsilon y_{3}+6 p^{2} y_{4}, \\
& \bar{y}_{2}=10 \epsilon y_{3}+6 p y_{4}, \\
& \bar{y}_{3}=6 y_{4}, \\
& \bar{y}_{4}=125 \epsilon^{3} y_{1}+75 p \epsilon^{2} y_{2}+30 p^{2} \epsilon y_{3}+\left(6 p^{3}-75 P_{3} \epsilon^{3}\right) y_{4},
\end{aligned}
$$

and where

$$
\epsilon^{3}=-\frac{3}{25 P_{3}}, \quad p=-\frac{125}{36}\left(8 P_{3}^{\prime}-5 P_{4}\right) \epsilon^{4},
$$

the development of the equations of the curve $C_{y}$ may be written in the canonical form

$$
\begin{aligned}
& Y=X^{2}+\Lambda_{7} X^{7}+\cdots \\
& Z=X^{3}+X^{6}+M_{7} X^{7}+\cdots,
\end{aligned}
$$

where $\Lambda_{7}, M_{7}, \ldots$ are given by (84) together with (81). This transformation is valid if $C_{y}$ does not belong to a linear complex.

The coefficients of $(86)$ are absolute invariants. We find, in fact,

$$
\Lambda_{7}=\frac{625 \epsilon^{5}}{7.12 \cdot 36 \theta_{3}}\left(25 \theta_{4}^{2}+20 \theta_{8}-4 \theta_{3.1}\right), \quad M_{7}=\frac{625 \epsilon^{4}}{42} \theta_{4},
$$

where

$$
\theta_{8}=4 \theta_{4} \theta_{3}^{\prime}-3 \theta_{3} \theta_{4}^{\prime} \text {. }
$$

From (86) and (76) it is clear that the plane $Y=0$ or $\bar{y}_{2}=0$ is the principal plane of the curve $C_{y}$ and its osculating cubic. In the original system of coördinates its equation will, therefore, be

$$
4 P_{3} x_{3}+\left(8 P_{3}^{\prime}-5 P_{4}\right) x_{4}=0 .
$$

If $C_{y}$ belongs to a linear complex, it coincides with the osculating plane.

The point which corresponds to (89) in the osculating linear complex is

$$
\left(5 P_{4}-8 P_{3}^{\prime}\right) y+4 P_{3} z,
$$

or in invariant form

$$
\left(\theta_{3}^{\prime}+\frac{5}{2} \theta_{4}\right) y+2 \theta_{3} z,
$$

Trans. Am. Math. Soc. 10 
an expression whose covariance may be verified directly. We shall call this point the principal point of the tangent. We thus obtain a curve on the developable of $C_{y}$ which may be called its principal curve.

If we make a transformation such that

$$
3 \eta=\theta_{3}^{\prime}+\frac{5}{2} \theta_{4},
$$

the point $P_{\bar{z}}$ describes the principal curve on the developable of $C_{y}$. The points $P_{\bar{\rho}}$ and $P_{\bar{\sigma}}$ also describe perfectly definite curves, whose expressions may be obtained from (9) by substituting for $\eta$ the expression (92). Any covariant may be expressed in terms of $y$ and the three which we have just determined, which may, therefore, serve to replace the covariants $C_{2}, C_{3}$ and $C_{4}$.

If $C_{y}$ belongs to a linear complex these four curves all coincide, so that a different set of fundamental covariants must then be selected.

In this exceptional case our fundamental tetrahedron $P_{y} P_{z} P_{\rho} P_{\sigma}$ gives rise to a most remarkable configuration. If we put again $P_{2}=0$, we have in this case also $P_{3}=0 . \quad P_{y} P_{\sigma}$ generates one of the developables of the third kind of which $C_{\sigma}$ is the cuspidal edge, while $P_{y} P_{z}$ of course generates the developable of which $C_{y}$ is the cuspidal edge. The surface generated by $P_{y} P_{\rho}$ is not developable. Its equations become

$$
\rho^{\prime \prime}+P_{4} y=0, \quad y^{\prime \prime}-\rho=0 .
$$

It belongs to the same linear complex as $C_{i j}$, and $C_{y}$ and $C_{\rho}$ are the two branches of its complex curve which is at the same time an asymptotic curve. $P_{z} P_{\rho}$ generates a developable, since $z^{\prime}=\rho$, of which $C_{z}$ is the cuspidal edge. $P_{\rho} P_{\sigma}$ generates a developable, since $\rho^{\prime}=\sigma$, of which $C_{\rho}$ is the cuspidal edge. Finally $P_{z} P_{\sigma}$ generates a ruled surface whose equations are

$$
\sigma^{\prime \prime}-P_{ \pm}^{\prime} \sigma_{t}^{\prime}+P_{4} z=0, \quad z^{\prime \prime}-\sigma=0,
$$

upon which $C_{z}$ and $C_{\sigma}$ are asymptotic lines.

We find, therefore, the following theorem.

If the curve belongs to a linear complex, we may, in an infinity of ways, choose the fundamental tetrahedron so that four of its edges give rise to developables whose cuspidal edges are described by the four vertices. The other two edges of the tetrahedron will then give rise to ruled surfaces upon each of which the vertices of the tetrahedron trace a pair of asymptotic curves. The latter coincide with the two branches of the complex curve for the derived surface of the second kind.

It does not seem convenient to develop the geometrical theory of the covariants for this case at the present moment, as it will appear naturally in connec- 
tion with some further investigations which I have not yet completed, and which I hope to present on some future occasion.

Those results which HALPHEN has deduced for the general case, and which have not been mentioned in this paper might now be directly transcribed from his memoir. For we have connected our own theory with his by obtaining the canonical form (86) for the development of the equations of the curve. In the case $\theta_{3}=0$, a different canonical form must be used, which has also been indicated by HaLPhen, but not extensively employed. The discussion of this we shall leave for a later occasion together with the investigations mentioned above.

Cambridge, England, June 20, 1904. 